\title{
Crosslinking of Extracellular Matrix Scaffolds Derived from Pluripotent Stem Cell Aggregates Modulates Neural Differentiation
}

\author{
Sébastien Sart ${ }^{1, \#}$, Yuanwei Yan ${ }^{1}$, Yan Li ${ }^{2,3}$, Eric Lochner ${ }^{4}$, \\ Changchun Zeng ${ }^{2,3}$, Teng $\mathrm{Ma}^{1}$, Yan $\mathrm{Li}^{1}{ }^{*}$
}

${ }^{1}$ Department of Chemical and Biomedical Engineering, FAMU-FSU College of Engineering, Florida State University, Tallahassee, Florida, USA

${ }^{2}$ Department of Industrial and Manufacturing Engineering, FAMU-FSU College of Engineering, Florida State University, Tallahassee, Florida, USA

${ }^{3}$ High-Performance Materials Institute, Florida State University, Tallahassee, Florida, USA

${ }^{4}$ Department of Physics, Florida State University, Tallahassee, Florida, USA

Running Title: Crosslinked PSC-ECM scaffolds regulate neural differentiation.

* Corresponding author:

Dr. Yan Li: address: 2525 Pottsdamer St., Tallahassee, FL 32310, Tel: 850-410-6320; Fax: 850410-6150; email: yli@eng.fsu.edu

${ }^{\#}$ Current address: Hydrodynamics Laboratory, CNRS UMR7646, Ecole Polytechnique, Palaiseau, France.

Submitted to Acta Biomaterialia 


\begin{abstract}
At various developmental stages, pluripotent stem cells (PSCs) and their progeny secrete a large amount of extracellular matrices (ECM) which could interact with regulatory growth factors to modulate stem cell lineage commitment. ECMs derived from PSC aggregates can be used as unique scaffolds that provide broad signaling capacities to mediate cellular differentiation. However, the rapid degradation of ECMs can impact their applications as the scaffolds for in vitro cell expansion and in vivo transplantation. To address this issue, this study investigated the effects of crosslinking on the ECMs derived from embryonic stem cells (ESCs) and the regulatory capacity of the crosslinked ECMs on the proliferation and differentiation of reseeded ESC-derived neural progenitor cells (NPCs). To create different biological cues, undifferentiated aggregates, spontaneous embryoid bodies, and ESC-derived NPC aggregates were decellularized. The derived ECMs were crosslinked using genipin or glutaraldehyde to enhance the scaffold stability. ESC-derived NPC aggregates were reseeded on different ECM scaffolds and differential cellular compositions of neural progenitors, neurons, and glial cells were observed. The results indicate that ESC-derived ECM scaffolds affect neural differentiation through intrinsic biological cues and biophysical properties. These scaffolds have potential for in vitro cell culture and in vivo tissue regeneration study.
\end{abstract}

Key words: Pluripotent stem cells, extracellular matrix, crosslinking, neural progenitor cells, decellularization 


\section{Introduction}

In recent years, pluripotent stem cells (PSCs), including induced pluripotent stem cells (iPSCs) and embryonic stem cells (ESCs), emerge as indefinite and non-invasive sources of neural progenitor cells (NPCs) due to their unique self-renewal ability and the capability of multi-lineage differentiation [1-3]. Although NPCs could be derived from the sub-ventricular zone and the hippocampus of adult and fetal brains, the limited cell number and the related ethical issues become major hurdles for clinical applications [4]. Alternatively, PSC-derived NPCs and neural tissues have shown promises for tissue engineering, organogenesis, drug screening, and disease modeling [1-3]. During neural tissue development from PSCs, extracellular matrix (ECM) plays a critical role in regulating cell survival, proliferation, and differentiation [5].

NPCs can secrete a large amount of ECMs and soluble factors which regulate cell proliferation and/or differentiation by autocrine feedback loop [6, 7]. Decellularized ECMs from tissues, organs, and in vitro cultured cells have been recently used as tissue engineering scaffolds or carriers for growth factor delivery [8-10]. For central nervous system repair, the decellularized ECMs derived from neural tissues have been demonstrated to possess neurosupportive functions $[11,12]$. Neural tissue-derived ECMs promote proliferation, migration, and neural differentiation of NPCs, possibly due to the presentation of adhesive ECM proteins and the sequestered neurotrophic factors (e.g., vascular endothelial growth factor, fibroblast growth factor (FGF)-2, and nerve growth factor) [13]. However, the use of these somatic cell-derived ECMs is limited by the availability of cell source.

PSCs are unlimited sources of cell-derived ECMs which can endogenously regulate the self-renewal and lineage commitment of stem cells [14-16]. These endogenous ECMs not only 
provide the cell adhesion sites, but also serve as the reservoirs for various exogenous and paracrine/autocrine factors secreted at different developmental stages, thus possessing the unique signaling capacity $[17,18]$. For instance, undifferentiated ESCs secrete various autocrine factors (i.e., Lefty, FGF-2) and ECMs (i.e., fibronectin) to regulate their self-renewal [19-21], while the ECMs derived from differentiated embryoid bodies (EBs) induce lineage-specific commitment $[15,16]$. Compared to adult tissue-derived ECMs, PSC-derived ECMs bear a broad spectrum of signaling molecules to regulate cellular differentiation and may partially recapitulate the microenvironment during tissue morphogenesis [22]. Recently, PSC-derived ECMs have been investigated as the scaffolds for cellular expansion and differentiation [23-25].

One critical issue for PSC-derived ECMs as scaffolds or carriers is their fast degradation (both in vivo and in vitro), which impacts their applications in regenerative medicine [26, 27]. To improve the ECM stability, modifications by crosslinking using genipin, glutaraldehyde or other methods have been explored for adult tissue-derived ECMs [26, 28]. Such modification would also increase the stiffness and change the biophysical properties of the ECM scaffolds [27]. The biophysical properties of various substrates or scaffolds were recently shown to differentially regulate lineage commitment of stem cells [29-31]. While PSC-derived ECMs have been assessed as tissue engineering scaffolds in several studies [15, 16, 32], the impact of crosslinked ECMs generated from PSCs at distinct developmental stages on neural differentiation has not been studied.

The objective of this study is to evaluate the crosslinking effects of ESC-derived ECMs on the scaffold stability and neural differentiation of the reseeded cells. Built on our previous work about ESC-derived ECM decellularization [16], the present study investigated the synergistic effects of the physical properties and the biochemical/biological properties of ESC- 
derived ECMs on neural differentiation through genipin-crosslinking [33, 34]. Genipin is a natural crosslinking reagent and is reported to have lower cytotoxicity than commonly used glutaraldehyde [35]. This study reveals the intricate interactions of ECM scaffolds with the reseeded cells during neural lineage commitment and is important for ECM scaffold design and functional characterizations.

\section{Materials and methods}

\subsection{Undifferentiated ESC cultures}

Murine ES-D3 line (American Type Culture Collection, Manassas, VA) was maintained on 6-well culture plates coated with $0.1 \%$ gelatin (Millipore, Temecula, CA) in a standard $5 \%$ $\mathrm{CO}_{2}$ incubator. The expansion medium was composed of Dulbecco's Modified Eagle's medium (DMEM, Invitrogen, Carlsbad, CA) supplemented with 10\% ESC-screened fetal bovine serum (FBS, Hyclone, Logan, UT), $1 \mathrm{mM}$ sodium pyruvate, $0.1 \mathrm{mM} \beta$-mercaptoethanol, penicillin (100 $\mathrm{U} / \mathrm{mL}$ ), streptomycin $(100 \mu \mathrm{g} / \mathrm{mL}$ ) (all from Invitrogen), and $1000 \mathrm{U} / \mathrm{mL}$ leukemia inhibitory factor (LIF, Millipore). The cells were seeded at $2-4 \times 10^{4}$ cells $/ \mathrm{cm}^{2}$ and sub-cultured every 2-3 days. This culture was used to generate undifferentiated aggregates, spontaneous EBs, and neural progenitor aggregates.

\subsection{Generation of Undifferentiated aggregates, EBs, and NPC aggregates}

Undifferentiated aggregates were obtained by seeding $1 \times 10^{6}$ cells from ESC monolayer into Ultra-Low Attachment (ULA) 6-well plates (Corning Incorporated, Corning, NY) in $3 \mathrm{~mL}$ growth media containing LIF [36]. The aggregates were cultivated for 3-4 days and used to derive ECM scaffolds. For spontaneous EB formation, $1 \times 10^{6}$ ESCs were seeded in ULA 6-well plates in $3 \mathrm{~mL}$ of differentiation medium. The differentiation medium consisted of DMEM supplemented with $10 \%$ FBS and $0.1 \mathrm{mM} \beta$-mercaptoethanol. The EBs were cultivated for 3-4 
days prior to decellularization. NPC aggregates were derived following the previously described procedure [37, 38]. Briefly, ESCs were seeded at $1 \times 10^{6}$ cells into ULA 6-well plates in $3 \mathrm{~mL}$ of DMEM-F12 plus 2\% B27 serum-free supplement (Invitrogen). At day 4, all-trans retinoic acid (RA, Sigma-Aldrich, St. Louis, MI) was supplemented at $1 \mu \mathrm{M}$ to enrich neural lineage. The cells were cultivated for additional 4 days and the NPC aggregates were collected to derive ECM scaffolds. The day 8 NPC aggregates were also used for seeding various ECM scaffolds. The aggregates were also formed in ULA 96-well plates $\left(3 \times 10^{4}\right.$ ESCs per well) to achieve uniform aggregate size for some study.

\subsection{Decellularization to generate ECM scaffolds}

The decellularization of ESC-derived aggregates was performed as previously described [16]. Briefly, about 600-1000 undifferentiated aggregates, EBs, or NPC aggregates were distributed into each of $1.5 \mathrm{~mL}$ microcentrifuge tubes and treated with $1 \%$ Triton X-100 (Sigma) for $30 \mathrm{~min}$. After the treatment, the samples were spun down at 18,000 g for $2 \mathrm{~min}$, rinsed with phosphate buffered saline (PBS), and incubated with 2,000 unit/mL DNAse I (Sigma) for 30 min. The samples were centrifuged at $18,000 \mathrm{~g}$ for $2 \mathrm{~min}$ again and rinsed with PBS prior to characterization or crosslinking.

\subsection{Crosslinking of ECM scaffolds}

Crosslinking of the ECM scaffolds was performed using genipin or glutaraldehyde [26]. Decellularized ECMs from undifferentiated aggregates (DE-A), EBs (DE-E), and NPC aggregates (DE-N) were incubated with 0.3\% genipin (Wako Chemicals USA, Inc, Richmond, VA) or $0.3 \%$ glutaraldehyde (Fisher scientific) for 6 hours. After the incubation, genipincrosslinked ECM scaffolds displayed blue color, which indicated the effective crosslinking as reported in literature $[26,35]$. The ECM scaffolds crosslinked with genipin $(\mathrm{G})$ were referred as DE-AG, DE-EG, and DE-NG. Similarly, the ECM scaffolds crosslinked with glutaraldehyde 
(GL) were referred as DE-AGL, DE-EGL, and DE-NGL (Table 1). All ECM scaffolds were rinsed six times in PBS prior to characterizations or reseeding with ESC-derived NPCs. The original non-crosslinked ECM scaffolds, denoted as DE-AC, DE-EC, and DE-NC, were used as the corresponding controls (C).

\subsection{Stability assay of ECM scaffolds}

The crosslinked or non-crosslinked ECM scaffolds were incubated with $100 \mu \mathrm{g} / \mathrm{mL}$ collagenase solution (Life Technologies) for 2, 4, or 6 hours. The morphology change of ECM scaffolds over time was imaged under a light microscope. The remaining ECM scaffolds after collagenase treatment were centrifuged at $800 \mathrm{~g}$ for $5 \mathrm{~min}$ and solubilized by sonication. The protein contents were then assessed by the Bradford protein assay (Bio-Rad) according to the manufacturer's instructions. The absorbance at $595 \mathrm{~nm}$ was read on a microplate reader (BioRad). The protein contents were normalized to the amount of proteins in the ECMs before collagenase treatment and this ratio was used as an indicator for the stability of ECM scaffolds.

\subsection{Scanning electron microscopy (SEM) and atomic force microscopy (AFM)}

For SEM observation, the ECM scaffolds were washed with PBS, fixed in $2.5 \%$ glutaraldehyde for 30-60 min and dehydrated in graded ethanol solutions. The samples were dried by hexamethyldisilazane (Sigma) evaporation, mounted, and sputter-coated with iridium. Observations were made using a Nova 400 Nano SEM (FEI, Hillsboro, OR) under low-vacuum conditions. For AFM analysis, the ECM samples were drop cast and dried on glass substrates. The surface roughness was measured by a Bruker Icon AFM (Digital Instruments, Santa Barbara, CA).

\subsection{Measurement of ECM scaffold modulus}

The Young's modulus of ECM scaffolds was measured using a dynamic mechanical analyzer (DMA Q800, TA Instruments) which has a force resolution of $0.00001 \mathrm{~N}$ and a strain 
resolution of $1 \mathrm{~nm}$. All tests were conducted at $30^{\circ} \mathrm{C}$ in compression model with a $0.0001 \mathrm{~N}$ preload and a strain rate of $0.1 \mathrm{~min}^{-1}$. The samples were trimmed from the bulk of hydrated decellularized ECM scaffolds to have a diameter of $5 \mathrm{~mm}$ and a thickness of $1 \mathrm{~mm}$, and mounted in a compression clamp with a diameter of $15 \mathrm{~mm}$. The Young's modulus was calculated from the slope of the initial linear portion $(<15 \%$ strain $)$ of the obtained stress-strain curve.

\subsection{NPC reseeding and cultivation on the decellularized ECMs}

For various ECM scaffolds, $1 \times 10^{6}$ dissociated NPCs or intact NPC aggregates derived from ESCs (in the passage range 20-35) were reseeded on the ECM scaffolds in ULA 6-well plates and cultivated for 3 days in DMEM-F12 containing 2\% B27 in the absence of growth factors. To study the effect of retinoid signaling, BMS 493 (1 $\mu \mathrm{M}$, Santa Cruz), a pan-retinoic acid receptor (RAR) antagonist, was supplemented in the media for some experiments. To evaluate cell seeding efficiency, NPC-ECM constructs were stained with $1 \mu \mathrm{M}$ calcein AM (Molecular Probes) and imaged under a fluorescent microscope (Olympus IX70, Melville, NY). The percentage of ECM scaffolds that were associated with NPC aggregates was assessed by dividing the number of scaffolds in contact with the aggregates against the total number of scaffolds. The size distributions of NPC aggregates and ECM scaffolds as well as the viability were analyzed by the ImageJ software. Due to the red auto fluorescence of the crosslinked ECMs [39], ethidium homodimer-1 staining was not used to determine cell viability (Supplementary Figure 1). The viability was calculated by dividing the green intensity (live cells stained with calcein AM) by the intensity of total cells (stained with 4',6-Diamidino-2Phenylindole (DAPI)). To determine the influence of ECM scaffolds on cell death, Image-iT ${ }^{\mathrm{TM}}$ Live Green Poly Caspase Detection kit (Molecular Probes) was used to detect caspase expression according to the manufacturer's instruction. To evaluate cell proliferation, cell numbers were 
determined using a hemocytometer after trypsin/EDTA dissociation. Fold expansion was calculated as the harvested cell number divided by the seeded cell number. Cell proliferation was further evaluated using bromodeoxyuridine (BrdU) assay. The samples were also incubated with $5 \mathrm{mg} / \mathrm{mL}$ 3-(4,5-Dimethylthiazol-2-yl)-2,5-diphenyltetrazolium bromide (MTT, Sigma) solution. Afterwards, the formazan crystals were hydrolyzed with dimethyl sulfoxide (Sigma) and measured at $500 \mathrm{~nm}$ using a microplate reader.

\subsection{BrdU assay}

Briefly, the cells were incubated in medium containing $10 \mu \mathrm{M}$ BrdU (Sigma) for $90 \mathrm{~min}$. The cells were then fixed with $70 \%$ cold ethanol, followed by a denaturation step using $2 \mathrm{~N}$ $\mathrm{HCl} / 0.5 \%$ Triton $\mathrm{X}-100$ for $30 \mathrm{~min}$ in the dark. The samples were reduced with $1 \mathrm{mg} / \mathrm{mL}$ sodium borohydride for 5 min and incubated with mouse anti-BrdU (1:100, Life Technologies) in blocking buffer $(0.5 \%$ Tween $20 / 1 \%$ bovine serum albumin in PBS), followed by Alexa Fluor ${ }^{\circledR} 488$ goat anti-Mouse $\operatorname{IgG}_{1}$ (Molecular Probes). The cells were counterstained with Hoechst 33342 and analyzed by a fluorescent microscope and the ImageJ software.

\subsection{Immunocytochemistry}

After 3 days in suspension, the NPC-ECM constructs were replated in 24-well plates coated with Geltrex ${ }^{\mathrm{TM}}$ (Life Technologies) for another 3 days. Neural differentiation was assessed by immunocytochemistry for neural markers. Briefly, the cells were fixed with $4 \%$ paraformaldehyde (PFA) and permeabilized with $0.2-0.5 \%$ Triton X-100. The samples were then blocked and incubated with mouse or rabbit primary antibody against: Nestin (Sigma) or Musashi-1 (Abcam) for neural progenitors, $\beta$-Tubulin III (Millipore) for neurons, or GFAP (Millipore) for astrocytes. After washing, the cells were incubated with the corresponding secondary antibody: Alexa Fluor ${ }^{\circledR} 488$ goat anti-Mouse $\operatorname{IgG}_{1}$ for GFAP and $\beta$-Tubulin III, or 
Alexa Fluor® 488 goat anti-Rabbit IgG for Nestin and Musashi-1 (Molecular Probes). The samples were then stained with Hoechst 33342 and visualized under a confocal microscope (Zeiss LSM 880).

\subsection{Flow cytometry}

To quantify neural marker expression, the cells on ECM scaffolds were harvested by trypsinization and analyzed by flow cytometry. Briefly, $1 \times 10^{6}$ cells per sample were fixed with 4\% PFA and washed with staining buffer (2\% FBS in PBS). The cells were permeabilized with $100 \%$ cold methanol, blocked, and then incubated with primary antibodies against Nestin, $\beta$ Tubulin III, GFAP, or Musashi-1 followed by the corresponding secondary antibody: Alexa Fluor® 488 goat anti-Mouse $\operatorname{IgG}_{1}$ (for GFAP and $\beta$-Tubulin III) or Alexa Fluor 488 goat antiRabbit IgG (for Nestin and Musashi-1). The cells were acquired with BD FACSCanto ${ }^{\text {TM }}$ II flow cytometer (Becton Dickinson) and analyzed against isotype controls using FlowJo software.

\subsection{Statistical analysis}

Each experiment was carried out at least three times. The average values of independent experiments were presented and the results are expressed as [mean \pm mean absolute deviation (MD)] unless otherwise noted. In each experiment, triplicate samples were used. To assess the statistical significance, ANOVA followed by Fisher's LSD post hoc tests were performed. A $p$ value $<0.05$ was considered statistically significant.

\section{Results}

\subsection{Characterization of decellularized ECM scaffolds}

DE-N scaffolds (i.e., ECMs derived from NPC aggregates) were used to characterize the effects of crosslinking with genipin (referred as G) on the physical properties of the decellularized ECMs. The ultrastructure analysis by SEM revealed changes in the morphology of ECM scaffolds upon crosslinking treatment. Non-crosslinked scaffolds (control, referred as 
C) displayed more porous structure while genipin-crosslinked scaffolds showed denser topography (Figure 1A). The roughness was lower for the non-crosslinked scaffolds compared to the crosslinked scaffolds ( $167 \pm 97 \mathrm{~nm} v s .284 \pm 30 \mathrm{~nm}$ or $331 \pm 67 \mathrm{~nm}$ ) as analyzed by AFM, but the difference was statistically insignificant $(p$-value $=0.15)$ (Figure 1B). Crosslinking increased the Young's modulus of ECM scaffolds from 4.9 $\pm 1.9 \mathrm{kPa}$ for the DE-NC group (noncrosslinked) to $13.9 \pm 0.7 \mathrm{kPa}$ for the DE-NG group (after crosslinking) according to the stress and stain curve (Figure 1C). In addition, stable scaffold size was observed for the DE-NG and DE-NGL (crosslinking with glutaraldehyde, referred as GL) scaffolds while the size of the DENC scaffolds decreased with collagenase treatment time (Figure 2A and Supplementary Figure 2). Consistently, the remaining protein content after collagenase treatment was $97 \%$ for the DE-NG group and $91 \%$ for the DE-NGL group, while the protein content decreased to $56 \%$ for the DE-NC group (Figure 2B). Together, crosslinking treatment increased the stiffness and structural stability of decellularized ECMs.

\subsection{Effects of the decellularized ECMs on NPC seeding and proliferation}

The different types of decellularized ECM scaffolds were seeded with intact ESC-derived NPC aggregates and the interactions of NPC aggregates with ECM scaffolds were assessed (Figure 3). NPC aggregates contacting ECM scaffolds were able to bridge with the scaffolds and some cells migrated and grew on the ECMs, especially for genipin-crosslinked scaffolds (Figure 3A). The crosslinking treatment by genipin was found to increase the percentage of scaffolds that were occupied with NPC aggregates compared to the non-crosslinked scaffolds (40-60\% vs. 5-30\%) (Figure 3B). Majority of the ECM scaffolds associated with the cells showed similar size range to the NPC aggregates (i.e., about $400 \mu \mathrm{m}$ ) (Figure 3C). 
Cell proliferation was evaluated by the fold increase after a 3-day culture on the scaffolds seeded with dissociated NPCs or intact NPC aggregates. Dissociated NPCs showed significantly lower expansion fold than intact NPC aggregates (0.5-1.0 vs. 1.2-2.5) for all the groups (Figure 4A). No significant difference was observed between the crosslinked and the non-crosslinked scaffolds in DE-A (i.e., ECMs derived from aggregates at undifferentiated state) or DE-E (i.e., ECMs derived from EBs) groups. The NPC aggregates reseeded on the DE-NC scaffolds had significantly lower expansion fold compared to other scaffolds (1.0 fold vs. 2.0-2.2 fold) except DE-NGL. The DE-NG scaffolds supported higher cell expansion fold than the DE-NC and DENGL groups, and was comparable to the DE-A and DE-E scaffolds (about 2.0-fold). Consistently, BrdU assay showed low percentage of positive cells for the DE-NC group (Table 2 and Supplementary Figure 3). MTT assays for intact NPC aggregates grown on the DE-A and DE-E scaffolds showed no significant difference for the crosslinked or non-crosslinked scaffolds, similar to the observation for expansion fold (Figure 4B). For DE-N scaffolds, cells in the DENG group had higher MTT activity compared to the DE-NC group at day 2 and 3, consistent with expansion fold data (Figure 4C). Cells in the DE-NGL group also showed higher MTT activity compared to the DE-NC group. Again, lower MTT activity was observed for dissociated NPCs compared to intact NPC aggregates. It was also notable that no significant difference in cell viability was observed for all the scaffolds (Figure 4D). The caspase expression showed no significant difference for different groups (Supplementary Figure 4). Due to better proliferation, intact NPC aggregates were used for the following experiments.

\subsection{Effects of decellularized ECMs on neural differentiation}

The expression of neural progenitor markers, Nestin and Musashi-1, was assessed for NPCs grown on all the scaffolds (Figure 5). Nestin expression was comparable for the cells 
grown on the crosslinked or the non-crosslinked scaffolds in DE-A and DE-E groups. However, for DE-N scaffolds, the DE-NC group showed lower Nestin expression $(61.0 \pm 5.8 \%$ vs. $76.3 \pm 3.3 \%$ ) than the DE-NG group while the difference compared to the DE-NGL group was not significant (Figure 5A-C). Musashi-1 expression was comparable for all DE-N scaffolds, while for DE-A groups, the DE-AGL group had lower Musashi-1 expression than the DE-AC and DEAG groups (65.1 $\pm 5.8 \%$ vs. $77-80 \%)$ (Figure 5D). Conversely, $\beta$-Tubulin III expression was comparable for the cells grown on the scaffolds in DE-A and DE-E groups, while the DE-NC group showed higher $\beta$-Tubulin III expression $(30.2 \pm 1.0 \%$ vs. $23 \pm 1.6 \%)$ than the DE-NG group (Figure 6). Thus, the DE-NC scaffolds enhanced neuronal differentiation compared to the DE-A and DE-E groups. Moreover, the crosslinking of DE-N scaffolds with genipin (i.e., DE-NG group) supported higher Nestin and lower $\beta$-Tubulin III expression than the DE-NC scaffolds. GFAP expression was enhanced for the DE-NC group compared to the DE-NG group (30.3 $\pm 8.3 \%$ vs. $12 \pm 0.1 \%)$, and for the DE-AC group compared to the DE-AG group $(28.0 \pm 1.0 \%$ vs. $12.3 \pm 2.3 \%$ ) (Figure 6D). The ratio of $\beta$-Tubulin $\mathrm{III}^{+}$cells to $\mathrm{GFAP}^{+}$cells was calculated and shown in Table 3. Genipin-crosslinking resulted in the increased ratio of $\beta$-Tubulin $\mathrm{III}^{+}$cells to $\mathrm{GFAP}^{+}$cells for DE-A and DE-N groups. The crosslinking effects using glutaraldehyde on neural differentiation were not as significant as genipin (except Musashi-1 and GFAP expressions in the DE-AGL group).

\subsection{Effects of retinoic acid-retinoic acid receptor $(\boldsymbol{R} A-R A R)$ signaling}

The DE-N scaffolds, derived from NPC aggregates through RA treatment, were more responsive to genipin-crosslinking treatment during neural differentiation. Therefore, the effects of RA-RAR signaling were investigated through +/- BMS 493 treatment for the DE-NC and DENG scaffolds (Figure 7). DE-AC and DE-EC scaffolds were included as controls. BMS 493 is 
a pan RAR antagonist that blocks RA-RAR interactions. It was observed that NPC treatment with BMS 493 reduced cell proliferation on the DE-EC and DE-NG scaffolds (Figure 7A). Nestin expression was increased upon BMS 493 treatment for the DE-EC and DE-NC groups (Figure 7B). Consistently, $\beta$-Tubulin III and GFAP expressions were decreased in cells grown on the DE-NC scaffolds (Figure 7C and 7D). BMS 493 treatment also increased GFAP expression for the DE-EC condition (Figure 7D). Together, these results indicated that the inhibition of RA-RAR signaling differentially modulated neural differentiation of NPCs on various types of ECM scaffolds.

\section{Discussions}

Dynamic interactions of acellular ECM scaffolds and stem cells are critical for lineagespecific commitment and tissue regeneration [40,41]. Understanding the synergistic effects of biochemical, biological, and biophysical properties of acellular ECM scaffolds would facilitate scaffold design and the functional regulation of stem cells [42-45]. The present study assessed the influence of crosslinked PSC-derived ECMs on neural differentiation and revealed the synergistic interactions of various ECM scaffold properties.

\subsection{Crosslinking of decellularized ECM scaffolds modulates neural differentiation}

Crosslinking improves the stability of ECM scaffolds and increases the elastic modulus, which affects the interactions of the cells with ECM scaffolds. The decellularized ECMs (after high speed centrifugation and condensation during decellularization) from ESC aggregates showed Young's modulus of $4.9 \mathrm{kPa}$, which is within the range (500 $\mathrm{Pa}-1.5 \mathrm{MPa}$ ) of various types of decellularized tissues and cells [27, 46, 47]. After crosslinking, the elastic modulus of ECM scaffolds was increased to $13.9 \mathrm{kPa}$. This crosslinking effect may regulate NPC seeding according to the differential interfacial tension hypothesis $[48,49]$. Based on this hypothesis, the 
cells tend to organize themselves according to cell-cortex tension, which results in the cells with low cell-cortex tension (softer) attach to the harder surface [48]. Because ECM crosslinking increases the scaffold's stiffness [50], the cells from NPC aggregates (softer) have higher tendency to associate with the crosslinked ECMs (harder) compared to the non-crosslinked ECMs (softer) (Figure 3A-b, 3A-d and 3A-f), leading to the enhanced reseeding of NPCs on the genipin-crosslinked ECM scaffolds.

The crosslinked ECM scaffolds can affect neural differentiation of ESC-derived NPCs and alter the populations of neuronal and glial cells due to the increased elastic modulus, especially for the DE-N groups in this study [51, 52]. Soft substrates (with elastic modulus of 1$7 \mathrm{kPa}$ ) have been reported to enhance neural differentiation of NPCs and PSCs compared to the substrates with higher elastic modulus (10 kPa to $1 \mathrm{GPa})[29,53,54]$. NPCs encapsulated in alginate hydrogels also showed gradual decrease of neuronal and glial differentiation when increasing the hydrogel modulus [55]. Consistently, in the present study the DE-NG group (higher modulus) had the lower percentages of neuronal cells (by 6\%) and glial cells (by 15\%) and the higher percentage of $\mathrm{Nestin}^{+}$cells (by 15\%) compared to the DE-NC group (lower modulus).

Crosslinking of decellularized ESC-ECM scaffolds enriches the proportion of neurons over astrocytes (Table 3), which could be attributed to the culture conditions that confound the effects of substrate stiffness on NPC fate decision. In the neuronal differentiation medium, increasing modulus from $1 \mathrm{kPa}$ to $1 \mathrm{GPa}$ was reported to have little effect on the percentage of neuronal cells [56], while glial cell purity was reduced during neural differentiation of adult neural stem cells [29]. In the culture medium that supported both neuronal and glial cells, stiffer surfaces were observed to reduce the percentage of neuronal cells while promoting glial cell 
population through the regulation of Rho GTPases $[29,53,57]$. In the present study, the retinol in B27 medium served as a source of RA and the differentiation medium promoted neuronal cell growth [58]. So, the increased stiffness via crosslinking had less effect on $\beta$-Tubulin $\mathrm{III}^{+}$cells, but reduced the percentage of $\mathrm{GFAP}^{+}$cells, resulting in the enriched proportion of neurons over astrocytes.

The effects of crosslinking by glutaraldehyde are less pronounced, but in similar trend to the crosslinking by genipin in general. Moreover, genipin is a natural crosslinking reagent which can result in low cytotoxicity [27]. So crosslinking with genipin is a preferred method.

\subsection{The aggregate properties prior to ECM decellularization modulates neural differentiation}

ECM crosslinking did not affect NPC proliferation and neuronal differentiation for the cells grown on the DE-A and DE-E scaffolds but affected the cells grown on the DE-N scaffolds, suggesting the synergistic effects of biophysical properties and biochemical/biological properties of ECMs. The differential cellular response can be attributed to both the heterogeneous populations of ESC-derived NPC aggregates and the properties of acellular ECM scaffolds.

ESC-derived NPC aggregates are heterogeneous and contain a mixture of primitive and definitive NPC populations [59, 60]. Primitive ESC-NPCs (mimicking neural precursors at E5.5-E7.5 of embryonic development) are LIF-dependent [61] and prone to endogenous RAmediated apoptosis [62]. In contrast, definitive NPCs generated from ESCs display the features of adult NPCs and are prone to neuronal and glial lineage commitment [63]. The response of heterogeneous NPC populations can be affected by the properties of ECM scaffolds.

The present study indicates that specific intrinsic cues exist for the three types of ECM scaffolds, i.e. DE-A, DE-E, and DE-N. The DE-NC scaffolds supported higher percentages of $\beta$ Tubulin $\mathrm{III}^{+}$cells and $\mathrm{GFAP}^{+}$cells and were influenced by genipin-crosslinking to a greater 
extent compared to the DE-AC and the DE-EC scaffolds. To elucidate the specific cues in different scaffolds, the treatment with BMS 493 to inhibit retinoid signaling was performed in this study. Our previous study indicated the possible role of retinoid signaling in the cells grown on the ECMs derived from RA-treated EBs (similar to the DE-N scaffolds) [16]. The RA-RAR regulation in retinoid signaling has recently been found as a critical pathway during neural tissue development $[64,65]$. For example, RA was reported to regulate NPC proliferation and GFAP expression of definitive NPCs through the activation of RAR $\alpha$ and RAR $\gamma$ [66]. By blocking RA-RAR interactions with BMS 493 (Figure 7), differential cellular responses to different ECM scaffolds revealed possible interactions of ECMs with exogenous or autocrine/paracrine factors.

It is postulated that the DE-AC scaffolds bound LIF during the culture of undifferentiated aggregates prior to decellularization [67]. When blocking the RA-RAR signaling, no changes in neural marker expression was observed for the DE-AC group, while the possible LIF bound to the DE-AC scaffolds would result in higher proliferation of NPCs [64]. DE-NC scaffolds (derived from NPC aggregates) may bind endogenous neurotrophic factors (i.e., NGF, BDNF, GDNF, FGF-2 etc.) that can enhance neural differentiation compared to the DE-EC and DE-AC scaffolds $[63,68]$. In addition, due to RA treatment before decellularization, cells grown on the DE-NC scaffolds contain higher retinoid signaling which can induce NPC differentiation towards neuronal and glial lineages [16, 69]. Therefore, by blocking RA-RAR interactions, neuronal and glial differentiations were reduced for the DE-NC group. DE-EC scaffolds, derived from spontaneous EBs, displayed less signaling specificity compared to the DE-AC and DE-NC scaffolds, but may still have endogenous retinoid signaling [70]. Conversely, the RARAR inhibition could favor the survival of primitive NPCs as indicated by the enhanced Nestin expression. Finally, NPCs reseeded on the DE-NG scaffolds showed little change in Nestin and 
GFAP expression but lower proliferation rate and $\beta$-Tubulin III expression with BMS 493 treatment, which was slightly different from the response for the cells grown on the DE-NC group. This observation indicates that the change in biophysical properties of ECMs due to crosslinking may affect their interactions with biological molecules, displaying the synergistic effects of various ECM properties.

PSC-derived ECMs are undefined materials containing numerous proteins and their characterization provides important information to elucidate the signaling molecules. Our previous study determined the key ECM proteins including fibronectin, laminin, Collagen IV and vitronectin [16]. For more detailed analysis, proteomics is a powerful tool to delineate the shared and distinct components in ECMs from different stages of stem cell development [71]. Our subsequent study uses proteomic analysis to characterize the three types of ECM scaffolds, i.e. DE-A, DE-E, and DE-N. DE-N group has higher spectra count of several laminin peptides and heparan sulfate core proteins (Yan et al, unpublished). It also has neurotropic proteins such as neuron-derived neurotrophic factor. These differences may partially explain the differences in cellular response to different types of ECMs. More in-depth analysis of cell-synthesized ECM microenvironment should provide further understanding on the natural signaling networks in PSC-derived ECMs and the associated growth factors to modulate stem cell fate decisions.

\section{Conclusion}

This study indicates that decellularized ECM scaffolds derived from different types of ESC aggregates display specific cues regulating the behavior of reseeded ESC-derived NPCs. Crosslinking modulates the structural and biophysical properties of ECM scaffolds, which enrich the neuronal cell population over the glial cell population. Decellularized ECM scaffolds derived from PSC aggregates at different developmental stages can regulate neural 
differentiation of the reseeded cells through the tunable biophysical and biological properties. The bioactive scaffolds derived from PSC-derived ECMs are suitable for the applications of in vitro cell culture. Since ECMs derived from murine ESCs cannot be used directly as the clinically relevant biomaterials in human, the following up step is to evaluate human induced pluripotent stem cell (hiPSC)-derived ECMs. The present study should help the further investigations on the ECM microenvironment generated from hiPSCs for in vivo applications.

\section{Acknowledgements}

The authors would like to thank Ms. Ruth Didier in FSU Department of Biomedical Sciences for her help in flow cytometry analysis and the analysis with Zeiss confocal microscopy. This work is supported by FSU start up fund, FSU Research Foundation GAP award, FSU COFRS award, and National Science Foundation (grant No. 1342192).

\section{Disclosures}

No competing financial interests exist.

\section{References:}

[1] Yu DX, Marchetto MC, Gage FH. Therapeutic translation of iPSCs for treating neurological disease. Cell Stem Cell. 2013;12:678-88.

[2] Engle SJ, Puppala D. Integrating human pluripotent stem cells into drug development. Cell Stem Cell. 2013;12:669-77.

[3] Lancaster MA, Renner M, Martin CA, Wenzel D, Bicknell LS, Hurles ME, et al. Cerebral organoids model human brain development and microcephaly. Nature. 2013;501:373-9.

[4] Gage FH. Mammalian neural stem cells. Science. 2000;287:1433-8.

[5] Solozobova V, Wyvekens N, Pruszak J. Lessons from the embryonic neural stem cell niche for neural lineage differentiation of pluripotent stem cells. Stem Cell Rev. 2012;8:813-29.

[6] Erlandsson A, Brannvall K, Gustafsdottir S, Westermark B, Forsberg-Nilsson K. Autocrine/paracrine platelet-derived growth factor regulates proliferation of neural progenitor cells. Cancer Res. 2006;66:8042-8. 
[7] Medberry CJ, Crapo PM, Siu BF, Carruthers CA, Wolf MT, Nagarkar SP, et al. Hydrogels derived from central nervous system extracellular matrix. Biomaterials. 2013;34:1033-40.

[8] Badylak SF, Taylor D, Uygun K. Whole-organ tissue engineering: decellularization and recellularization of three-dimensional matrix scaffolds. Annu Rev Biomed Eng. 2011;13:27-53.

[9] Seif-Naraghi SB, Horn D, Schup-Magoffin PJ, Christman KL. Injectable extracellular matrix derived hydrogel provides a platform for enhanced retention and delivery of a heparin-binding growth factor. Acta Biomater. 2012;8:3695-703.

[10] DeQuach JA, Yuan SH, Goldstein LS, Christman KL. Decellularized porcine brain matrix for cell culture and tissue engineering scaffolds. Tissue Eng Part A. 2011;17:2583-92.

[11] Volpato FZ, Fuhrmann T, Migliaresi C, Hutmacher DW, Dalton PD. Using extracellular matrix for regenerative medicine in the spinal cord. Biomaterials. 2013;34:4945-55.

[12] Crapo PM, Tottey S, Slivka PF, Badylak SF. Effects of biologic scaffolds on human stem cells and implications for CNS tissue engineering. Tissue Eng Part A. 2014;20:313-23.

[13] Crapo PM, Medberry CJ, Reing JE, Tottey S, van der Merwe Y, Jones KE, et al. Biologic scaffolds composed of central nervous system extracellular matrix. Biomaterials. 2012;33:353947.

[14] Nair R, Ngangan AV, Kemp ML, McDevitt TC. Gene expression signatures of extracellular matrix and growth factors during embryonic stem cell differentiation. PLoS One. 2012;7:e42580.

[15] Goh SK, Olsen P, Banerjee I. Extracellular matrix aggregates from differentiating embryoid bodies as a scaffold to support ESC proliferation and differentiation. PLoS One. 2013;8:e61856.

[16] Sart S, Ma T, Li Y. Extracellular matrices decellularized from embryonic stem cells maintained their structure and signaling specificity. Tissue Eng Part A 2014;20:54-66.

[17] Badylak SF. The extracellular matrix as a biologic scaffold material. Biomaterials. 2007;28:3587-93.

[18] Hynes RO. The extracellular matrix: not just pretty fibrils. Science. 2009;326:1216-9.

[19] Przybyla LM, Voldman J. Attenuation of extrinsic signaling reveals the importance of matrix remodeling on maintenance of embryonic stem cell self-renewal. Proc Natl Acad Sci U S A. 2012;109:835-40.

[20] Hunt GC, Singh P, Schwarzbauer JE. Endogenous production of fibronectin is required for self-renewal of cultured mouse embryonic stem cells. Exp Cell Res. 2012;318:1820-31. 
[21] Hughes C, Radan L, Chang WY, Stanford WL, Betts DH, Postovit LM, et al. Mass spectrometry-based proteomics analysis of the matrix microenvironment in pluripotent stem cell culture. Mol Cell Proteomics. 2012;12:1924-36.

[22] Fitzpatrick LE, McDevitt TC. Cell-derived matrices for tissue engineering and regenerative medicine applications Biomater Sci. 2015;3:12-24.

[23] Ngangan AV, McDevitt TC. Acellularization of embryoid bodies via physical disruption methods. Biomaterials. 2009;30:1143-9.

[24] Rodrigues CA, Diogo MM, da Silva CL, Cabral JM. Microcarrier expansion of mouse embryonic stem cell-derived neural stem cells in stirred bioreactors. Biotechnol Appl Biochem. 2011;58:231-42.

[25] Sart S, Agathos SN, Li Y. Engineering stem cell fate with biochemical and biomechanical properties of microcarriers. Biotechnol Prog. 2013;29:1354-66.

[26] Liang HC, Chang Y, Hsu CK, Lee MH, Sung HW. Effects of crosslinking degree of an acellular biological tissue on its tissue regeneration pattern. Biomaterials. 2004;25:3541-52.

[27] Jiang T, Ren XJ, Tang JL, Yin H, Wang KJ, Zhou CL. Preparation and characterization of genipin-crosslinked rat acellular spinal cord scaffolds. Mater Sci Eng C Mater Biol Appl. 2013;33:3514-21.

[28] Lee JH, Kim HG, Lee WJ. Characterization and tissue incorporation of cross-linked human acellular dermal matrix. Biomaterials. 2015;44:195-205.

[29] Saha K, Keung AJ, Irwin EF, Li Y, Little L, Schaffer DV, et al. Substrate modulus directs neural stem cell behavior. Biophys J. 2008;95:4426-38.

[30] Musah S, Wrighton PJ, Zaltsman Y, Zhong X, Zorn S, Parlato MB, et al. Substratuminduced differentiation of human pluripotent stem cells reveals the coactivator YAP is a potent regulator of neuronal specification. Proc Natl Acad Sci U S A. 2014;111:13805-10.

[31] Zoldan J, Karagiannis ED, Lee CY, Anderson DG, Langer R, Levenberg S. The influence of scaffold elasticity on germ layer specification of human embryonic stem cells. Biomaterials. 2011;32:9612-21.

[32] Nair R, Shukla S, McDevitt TC. Acellular matrices derived from differentiating embryonic stem cells. J Biomed Mater Res A. 2008;87:1075-85.

[33] Qiu J, Li J, Wang G, Zheng L, Ren N, Liu H, et al. In vitro investigation on the biodegradability and biocompatibility of genipin cross-linked porcine acellular dermal matrix with intrinsic fluorescence. ACS Appl Mater Interfaces. 2013;5:344-50. 
[34] Liu Z, Zhou Q, Zhu J, Xiao J, Wan P, Zhou C, et al. Using genipin-crosslinked acellular porcine corneal stroma for cosmetic corneal lens implants. Biomaterials. 2012;33:7336-46.

[35] Butler MF, Ng Y-F, Pudney PDA. Mechanism and kinetics of the crosslinking reaction between biopolymers containing primary amine groups and Genipin. J. Polym. Sci., Part A: Polym. Chem. 2003;41:3941-53.

[36] Sart S, Ma T, Li Y. Cryopreservation of pluripotent stem cell aggregates in defined proteinfree formulation. Biotechnol Prog. 2013;29:143-53.

[37] Sart S, Liu Y, Ma T, Li Y. Microenvironment regulation of pluripotent stem cell-derived neural progenitor aggregates by human mesenchymal stem cell secretome. Tissue Eng Part A. 2014;20:2666-79.

[38] Sart S, Calixto Bejarano F, Baird MA, Yan Y, Rosenberg JT, Ma T, et al. Intracellular labeling of mouse embryonic stem cell-derived neural progenitor aggregates with micron-sized particles of iron oxide. Cytotherapy. 2015;17:98-111.

[39] Hwang YJ, Larsen J, Krasieva TB, Lyubovitsky JG. Effect of genipin crosslinking on the optical spectral properties and structures of collagen hydrogels. ACS Appl Mater Interfaces. 2011;3:2579-84.

[40] Prewitz MC, Seib FP, von Bonin M, Friedrichs J, Stissel A, Niehage C, et al. Tightly anchored tissue-mimetic matrices as instructive stem cell microenvironments. Nat Methods. 2013;10:788-94.

[41] Lu TY, Lin B, Kim J, Sullivan M, Tobita K, Salama G, et al. Repopulation of decellularized mouse heart with human induced pluripotent stem cell-derived cardiovascular progenitor cells. Nat Commun. 2013;4:2307.

[42] Celiz AD, Smith JG, Langer R, Anderson DG, Winkler DA, Barrett DA, et al. Materials for stem cell factories of the future. Nat Mater. 2014;13:570-9.

[43] Kothapalli CR, Kamm RD. 3D matrix microenvironment for targeted differentiation of embryonic stem cells into neural and glial lineages. Biomaterials. 2013;34:5995-6007.

[44] O'Neill JD, Freytes DO, Anandappa AJ, Oliver JA, Vunjak-Novakovic GV. The regulation of growth and metabolism of kidney stem cells with regional specificity using extracellular matrix derived from kidney. Biomaterials. 2013;34:9830-41.

[45] Vunjak-Novakovic G, Scadden DT. Biomimetic platforms for human stem cell research. Cell Stem Cell. 2011;8:252-61.

[46] Choi JS, Kim BS, Kim JY, Kim JD, Choi YC, Yang HJ, et al. Decellularized extracellular matrix derived from human adipose tissue as a potential scaffold for allograft tissue engineering. J Biomed Mater Res A. 2011;97:292-9. 
[47] Xing Q, Vogt C, Leong KW, Zhao F. Highly aligned nanofibrous scaffold derived from decellularized human fibroblasts. Adv Funct Mater. 2014;24:3027-35.

[48] Krieg M, Arboleda-Estudillo Y, Puech PH, Kafer J, Graner F, Muller DJ, et al. Tensile forces govern germ-layer organization in zebrafish. Nat Cell Biol. 2008;10:429-36.

[49] Maitre JL, Berthoumieux H, Krens SF, Salbreux G, Julicher F, Paluch E, et al. Adhesion functions in cell sorting by mechanically coupling the cortices of adhering cells. Science. 2012;338:253-6.

[50] Youngstrom DW, Barrett JG, Jose RR, Kaplan DL. Functional characterization of detergent-decellularized equine tendon extracellular matrix for tissue engineering applications. PLoS One. 2013;8:e64151.

[51] Discher DE, Janmey P, Wang YL. Tissue cells feel and respond to the stiffness of their substrate. Science. 2005;310:1139-43.

[52] Sun Y, Yong KM, Villa-Diaz LG, Zhang X, Chen W, Philson R, et al. Hippo/YAPmediated rigidity-dependent motor neuron differentiation of human pluripotent stem cells. Nat Mater. 2014;13:599-604.

[53] Leipzig ND, Shoichet MS. The effect of substrate stiffness on adult neural stem cell behavior. Biomaterials. 2009;30:6867-78.

[54] Keung AJ, Asuri P, Kumar S, Schaffer DV. Soft microenvironments promote the early neurogenic differentiation but not self-renewal of human pluripotent stem cells. Integr Biol (Camb). 2012;4:1049-58.

[55] Banerjee A, Arha M, Choudhary S, Ashton RS, Bhatia SR, Schaffer DV, et al. The influence of hydrogel modulus on the proliferation and differentiation of encapsulated neural stem cells. Biomaterials. 2009;30:4695-9.

[56] Keung AJ, Dong M, Schaffer DV, Kumar S. Pan-neuronal maturation but not neuronal subtype differentiation of adult neural stem cells is mechanosensitive. Sci Rep. 2013;3:1817.

[57] Keung AJ, de Juan-Pardo EM, Schaffer DV, Kumar S. Rho GTPases mediate the mechanosensitive lineage commitment of neural stem cells. STEM CELLS. 2011;29:1886-97.

[58] Engberg N, Kahn M, Petersen DR, Hansson M, Serup P. Retinoic acid synthesis promotes development of neural progenitors from mouse embryonic stem cells by suppressing endogenous, Wnt-dependent nodal signaling. STEM CELLS. 2010;28:1498-509.

[59] Li M, Pevny L, Lovell-Badge R, Smith A. Generation of purified neural precursors from embryonic stem cells by lineage selection. Curr Biol. 1998;8:971-4. 
[60] Cai C, Grabel L. Directing the differentiation of embryonic stem cells to neural stem cells. Dev Dyn. 2007;236:3255-66.

[61] Tropepe V, Hitoshi S, Sirard C, Mak TW, Rossant J, van der Kooy D. Direct neural fate specification from embryonic stem cells: a primitive mammalian neural stem cell stage acquired through a default mechanism. Neuron. 2001;30:65-78.

[62] Herget T, Esdar C, Oehrlein SA, Heinrich M, Schutze S, Maelicke A, et al. Production of ceramides causes apoptosis during early neural differentiation in vitro. $J$ Biol Chem. 2000;275:30344-54.

[63] Rowland JW, Lee JJ, Salewski RP, Eftekharpour E, van der Kooy D, Fehlings MG. Generation of neural stem cells from embryonic stem cells using the default mechanism: in vitro and in vivo characterization. Stem Cells Dev. 2011;20:1829-45.

[64] Goncalves MB, Boyle J, Webber DJ, Hall S, Minger SL, Corcoran JP. Timing of the retinoid-signalling pathway determines the expression of neuronal markers in neural progenitor cells. Dev Biol. 2005;278:60-70.

[65] Stergiopoulos A, Politis PK. The role of nuclear receptors in controlling the fine balance between proliferation and differentiation of neural stem cells. Arch Biochem Biophys. 2013;534:27-37.

[66] Yu S, Levi L, Siegel R, Noy N. Retinoic acid induces neurogenesis by activating both retinoic acid receptors (RARs) and peroxisome proliferator-activated receptor beta/delta (PPARbeta/delta). J Biol Chem. 2012;287:42195-205.

[67] Mereau A, Grey L, Piquet-Pellorce C, Heath JK. Characterization of a binding protein for leukemia inhibitory factor localized in extracellular matrix. J Cell Biol. 1993;122:713-9.

[68] Martino G, Pluchino S, Bonfanti L, Schwartz M. Brain regeneration in physiology and pathology: the immune signature driving therapeutic plasticity of neural stem cells. Physiol Rev. 2011;91:1281-304.

[69] Santos T, Ferreira R, Maia J, Agasse F, Xapelli S, Cortes L, et al. Polymeric nanoparticles to control the differentiation of neural stem cells in the subventricular zone of the brain. ACS Nano. 2012;6:10463-74.

[70] Simandi Z, Balint BL, Poliska S, Ruhl R, Nagy L. Activation of retinoic acid receptor signaling coordinates lineage commitment of spontaneously differentiating mouse embryonic stem cells in embryoid bodies. FEBS Lett. 2010;584:3123-30.

[71] Byron A, Humphries JD, Humphries MJ. Defining the extracellular matrix using proteomics. Int J Exp Pathol. 2013;94:75-92. 
Table 1. A list of decellularized crosslinked and non-crosslinked ECMs derived from ESCs.

\begin{tabular}{|c|c|c|c|}
\hline Abbreviation & $\begin{array}{l}\text { ESC growth } \\
\text { condition }\end{array}$ & Crosslinking & $\begin{array}{l}\text { Growth factor } \\
\text { treatment }\end{array}$ \\
\hline DE-AC & $\begin{array}{l}\text { Undifferentiated } \\
\text { aggregates }\end{array}$ & None & LIF \\
\hline DE-AG & $\begin{array}{l}\text { Undifferentiated } \\
\text { aggregates }\end{array}$ & Genipin & LIF \\
\hline DE-AGL & $\begin{array}{l}\text { Undifferentiated } \\
\text { aggregates }\end{array}$ & Glutaraldehyde & LIF \\
\hline DE-EC & $\begin{array}{l}\text { Spontaneously } \\
\text { differentiated EBs }\end{array}$ & None & None \\
\hline DE-EG & $\begin{array}{l}\text { Spontaneously } \\
\text { differentiated EBs }\end{array}$ & Genipin & None \\
\hline DE-EGL & $\begin{array}{l}\text { Spontaneously } \\
\text { differentiated EBs }\end{array}$ & Glutaraldehyde & None \\
\hline DE-NC & NPC aggregates & None & RA \\
\hline DE-NG & NPC aggregates & Genipin & RA \\
\hline DE-NGL & NPC aggregates & Glutaraldehyde & RA \\
\hline
\end{tabular}

ECM: extracellular matrix. ESC: embryonic stem cell. LIF: leukemia inhibitory factor. RA: Retinoic acid. NPC: neural progenitor cells 
Table 2. BrdU ${ }^{+}$cells in the outgrowth of the replated ESC-NPCs grown on various ECM scaffolds.

\begin{tabular}{cc}
\hline Scaffolds & BrdU $^{+}$cells $(\%)$ \\
\hline DE-AC & $38.7 \pm 13.8$ \\
DE-AG $^{\text {a }}$ & $40.1 \pm 2.8$ \\
DE-AGL & $33.8 \pm 9.7$ \\
\hline DE-EC & \\
DE-EG & $30.1 \pm 3.7$ \\
DE-EGL & $30.2 \pm 7.0$ \\
\hline DE-NC & $31.6 \pm 7.0$ \\
\hline DE-NG & $22.2 \pm 4.1$ \\
DE-NGL $^{\text {a }}$ & $43.1 \pm 9.7$ \\
\hline
\end{tabular}

${ }^{a}$ indicates statistical difference $(p$-value $<0.05)$ compared to the DE-NC group. ${ }^{\mathrm{b}}$ indicates statistical difference $(p$-value $<0.05)$ compared to the DE-NG group. 
Table 3. The ratio of $\beta$-Tubulin III $^{+}$cells to GFAP $^{+}$cells in various ECM scaffolds.

\begin{tabular}{cc}
\hline Scaffolds & Ratio of $\boldsymbol{\beta}$-Tubulin III $^{+} /$GFAP $^{+}$ \\
\hline DE-AC & $0.9 \pm 0.1$ \\
DE-AG $^{\mathrm{a}}$ & $2.3 \pm 0.7$ \\
DE-AGL $^{\mathrm{a}}$ & $3.4 \pm 0.9$ \\
\hline DE-EC & $1.5 \pm 0.4$ \\
DE-EG & $2.0 \pm 0.5$ \\
DE-EGL & $1.5 \pm 0.5$ \\
\hline DE-NC & $1.1 \pm 0.2$ \\
DE-NG & $1.9 \pm 0.1$ \\
DE-NGL & $1.7 \pm 0.8$
\end{tabular}

${ }^{a}$ indicates statistical difference $(p$-value $<0.05)$ compared to the corresponding control $(\mathrm{C})$ scaffolds. 


\section{Figure Legends}

Figure 1. Ultrastructure and biophysical properties of the non-crosslinked and crosslinked ECM scaffolds. (A) Ultrastructure of ECM scaffolds assessed by scanning electron microscopy (SEM): (i) and (iii) non-crosslinked; (ii) and (iv) genipin-crosslinked. (i) and (ii) are at 2000X; (iii) and (iv) are at 500X. (B) Representative atomic force microscopy (AFM) images (i-iii) and the quantification of surface roughness for non-crosslinked and crosslinked ECMs (iv); (C) The stress-strain curves $(n=3)$ for the quantification of elastic modulus of (i) non-crosslinked and (ii) genipin-crosslinked ECMs.

Figure 2. Crosslinking enhanced the stability of ECM scaffolds. DE-NC, DE-NG and DENGL were treated with collagenase for 2, 4, and 6 hours. (A) Representative morphology of the ECM scaffolds upon collagenase treatment. Scale bar: $100 \mu \mathrm{m}$. (B) Quantification of the percentage of remaining proteins from the DE-NC, DE-NG, and DE-NGL scaffolds after collagenase treatment. a, b, and c indicate $p$-value $<0.05$, which show the significant differences (DE-NG vs. DE-NC, or DE-NGL vs. DE-NC) at different time points (a for 2-hour point, b for 4-hour point, and c for 6-hour point).

Figure 3. Morphology of the reseeded ESC-NPCs on the crosslinked and non-crosslinked ECM scaffolds. (A) Representative images of reseeded NPCs (stained with calcein AM to show live cells) on the decellularized scaffolds. Long arrows point to the non-crosslinked ECM scaffolds, which are highlighted with white circle. Short arrows point to the crosslinked ECM scaffolds displaying red auto fluorescence. Scale bar: $100 \mu \mathrm{m}$. (B) Percentage of ECM scaffolds associated with the cells. (C) Size distribution of NPC aggregates and the ECM scaffolds interacting with NPCs. * $p$-value $<0.05$.

Figure 4. Proliferation and viability of the reseeded ESC-NPCs grown on different ECM scaffolds. (A) Fold increase in cell number at day 3 after reseeding; (B) MTT activity for NPCs reseeded on DE-A and DE-E scaffolds; Results from a representative experiment were presented here. (C) MTT activity for dissociated and intact NPCs reseeded on the DE-NC, DE-NG and DE-NGL scaffolds. a indicated significant difference between the intact and dissociated cells for 
DE-NGL, $b$ was for the intact and dissociated cells for DE-NG, and c was for DE-NC; \# indicated significant difference among the various scaffolds (DE-NC, DE-NG, DE-NGL) for the cultures of intact aggregates. Results from a representative experiment were presented here. (D) Viability of intact NPCs reseeded on decellularized ECM scaffolds. The relative viability was calculated by dividing the green intensity of live cells by the blue intensity of the total cells analyzed by ImageJ software. $* p$-value $<0.05$.

Figure 5. Expression of neural progenitor markers of the reseeded ESC-NPCs grown on different ECM scaffolds. (A) Representative confocal fluorescent images of Nestin and Musashi-1 expression; Green: Nestin or Musashi-1; Blue: Hoechst 33342. Scale bar: $100 \mu \mathrm{m}$. (B) Representative flow cytometry histograms of Nestin and Musashi-1 expression. Percentage of the cells positive for Nestin. (D) Percentage of the cells positive for Musashi-1. * $p$-value $<0.05$.

Figure 6. Neural differentiation of the reseeded ESC-NPCs grown on different ECM scaffolds. (A) Representative confocal fluorescent images of $\beta$-Tubulin III and GFAP expression; Green: $\beta$-Tubulin III or GFAP; Blue: Hoechst 33342. Scale bar: $100 \mu \mathrm{m}$. Representative flow cytometry histograms of $\beta$-Tubulin III and GFAP expression. (C) Percentage of the cells positive for $\beta$-Tubulin III. (D) Percentage of the cells positive for GFAP. $* p$-value $<0.05$.

Figure 7. Effects of BMS 493 treatment on the cells grown on different ECM scaffolds. (A) Fold change in the proliferation of the reseeded ESC-NPCs after BMS 493 treatment (+BMS) compared to untreated cultures (-BMS). Fold change in the expression of (B) Nestin, (C) $\beta$ Tubulin III, and (D) GFAP. The fold change was calculated as the ratio of the expression after BMS 493 treatment (+BMS) compared to that of untreated cultures (-BMS). * $p$-value $<0.05$. 


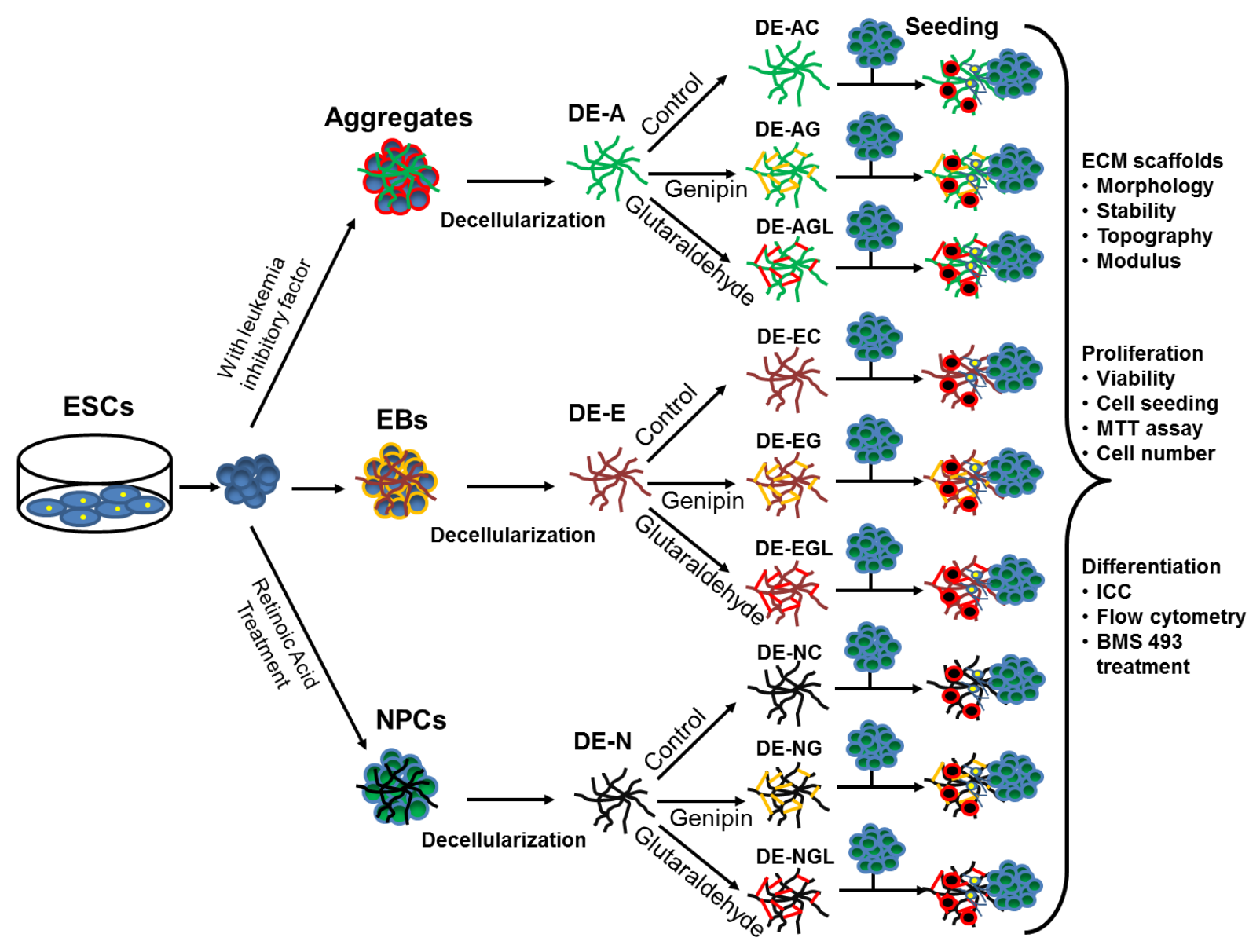

Graphical Abstract: Schematic diagram of the experiments. Embryonic stem cells (ESCs) were seeded in suspension to generate undifferentiated aggregates (AGG), spontaneous embryoid bodies (EBs), or to derive neural progenitor cell (NPC) aggregates. The three types of aggregates were then decellularized (DE) to generate DE-A, DE-E, and DE-N scaffolds. The derived extracellular matrices (ECMs) were treated with genipin $(\mathrm{G})$ or glutaraldehyde (GL) to obtain the crosslinked scaffolds. The non-crosslinked ECMs were used as controls (C). ESCderived NPCs were seeded onto various ECM scaffolds and characterized. 

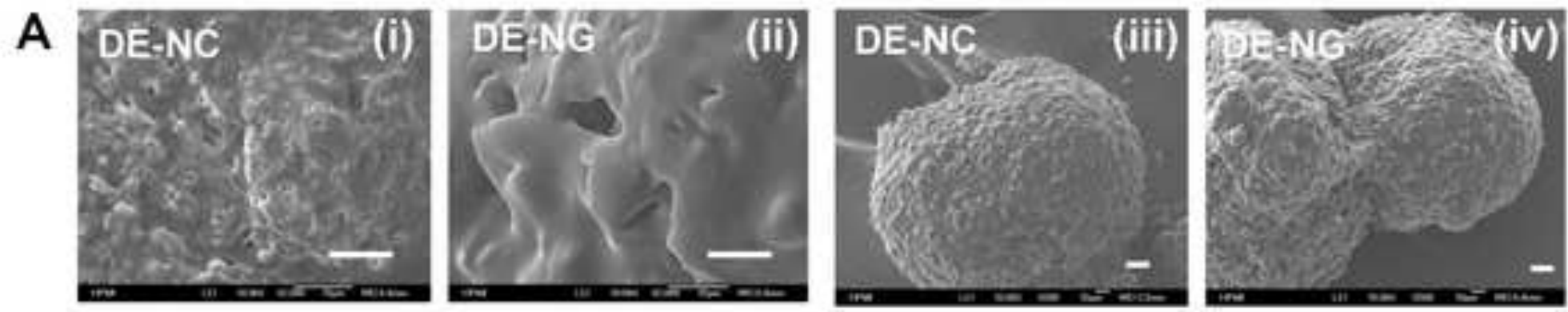

B
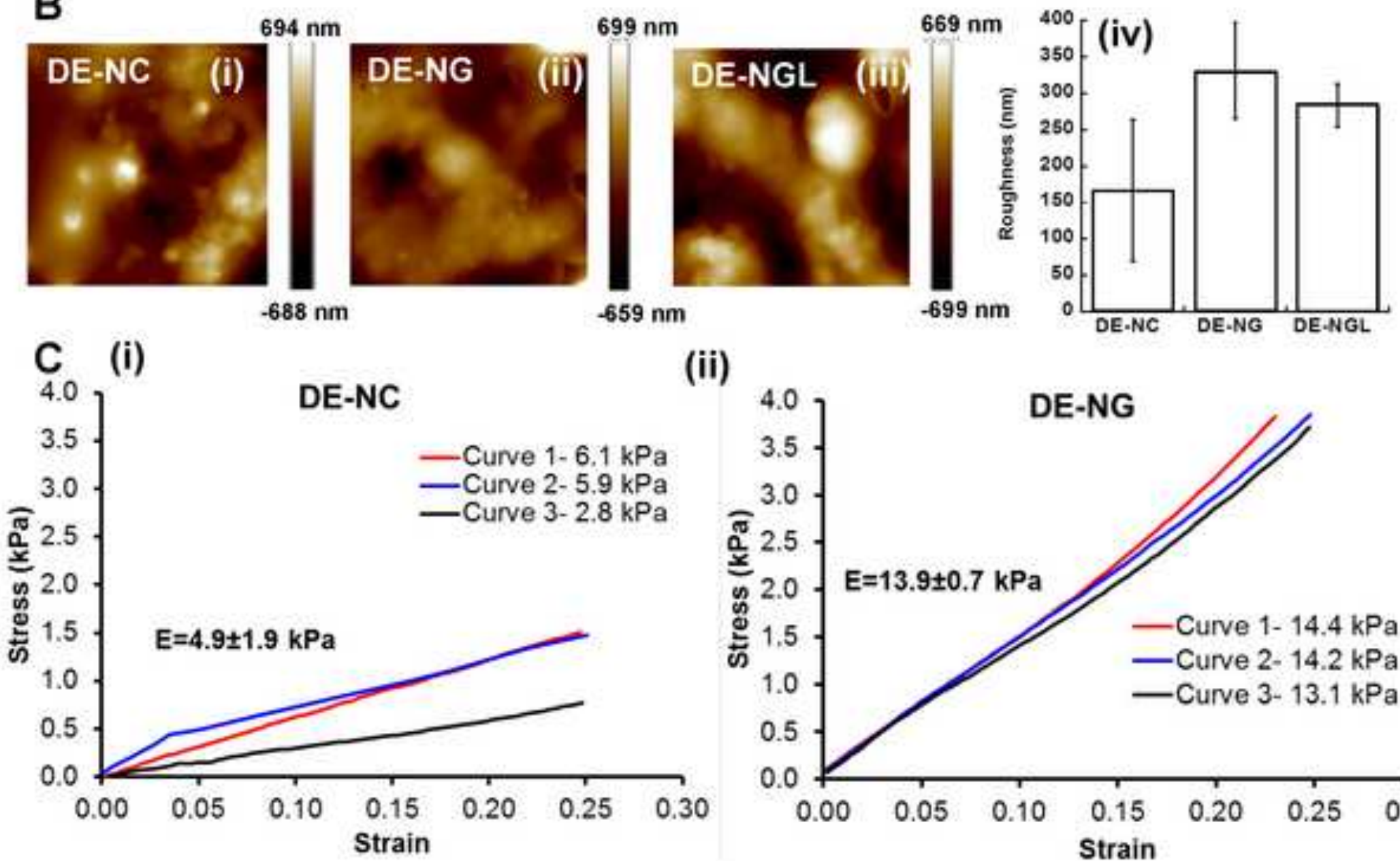

(ii)

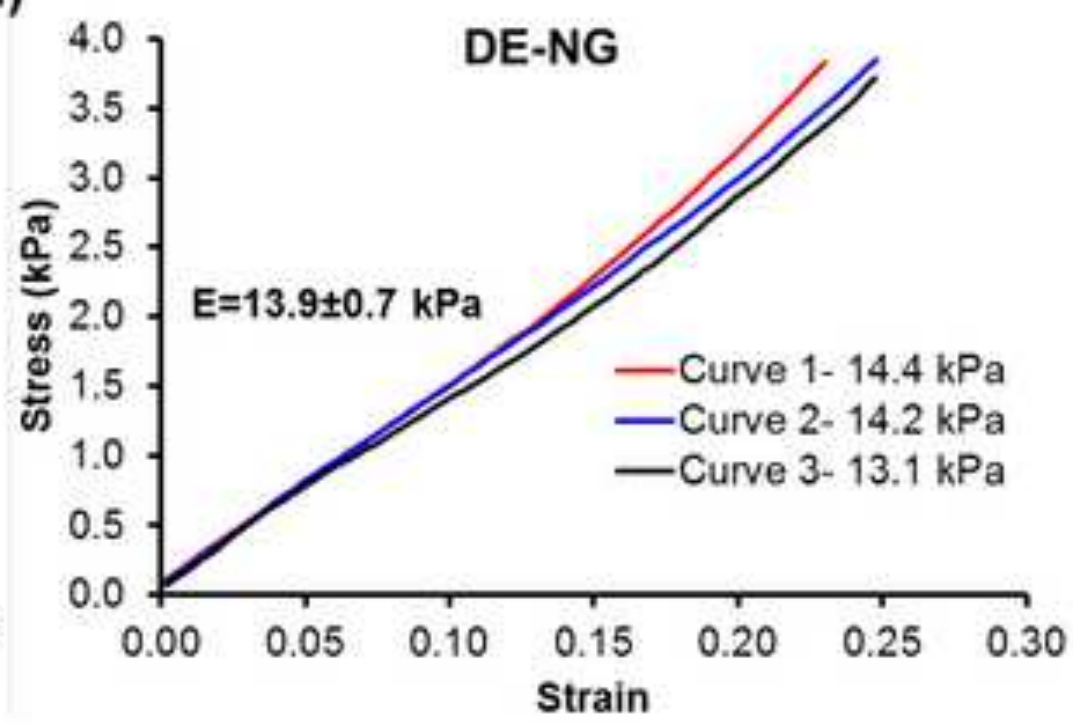


A

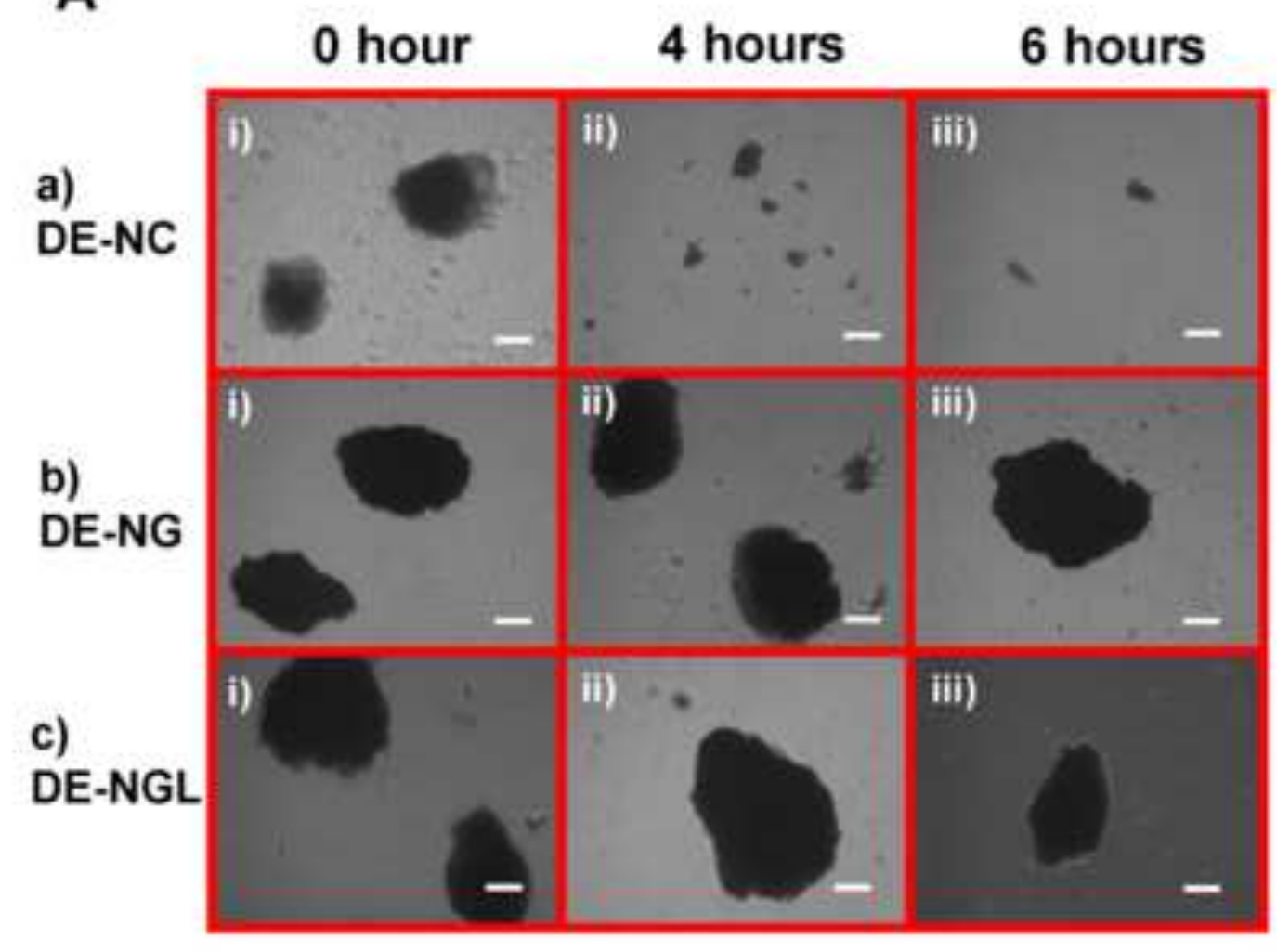

B

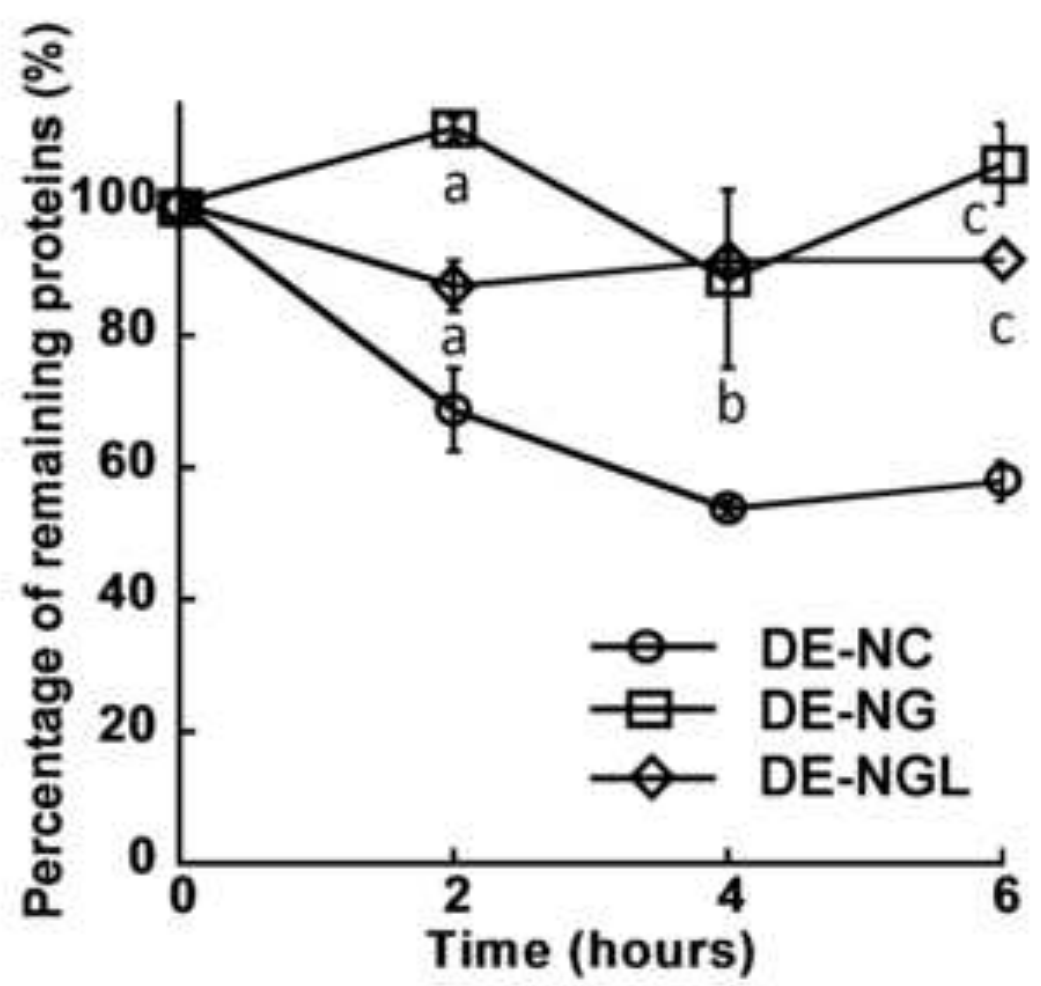



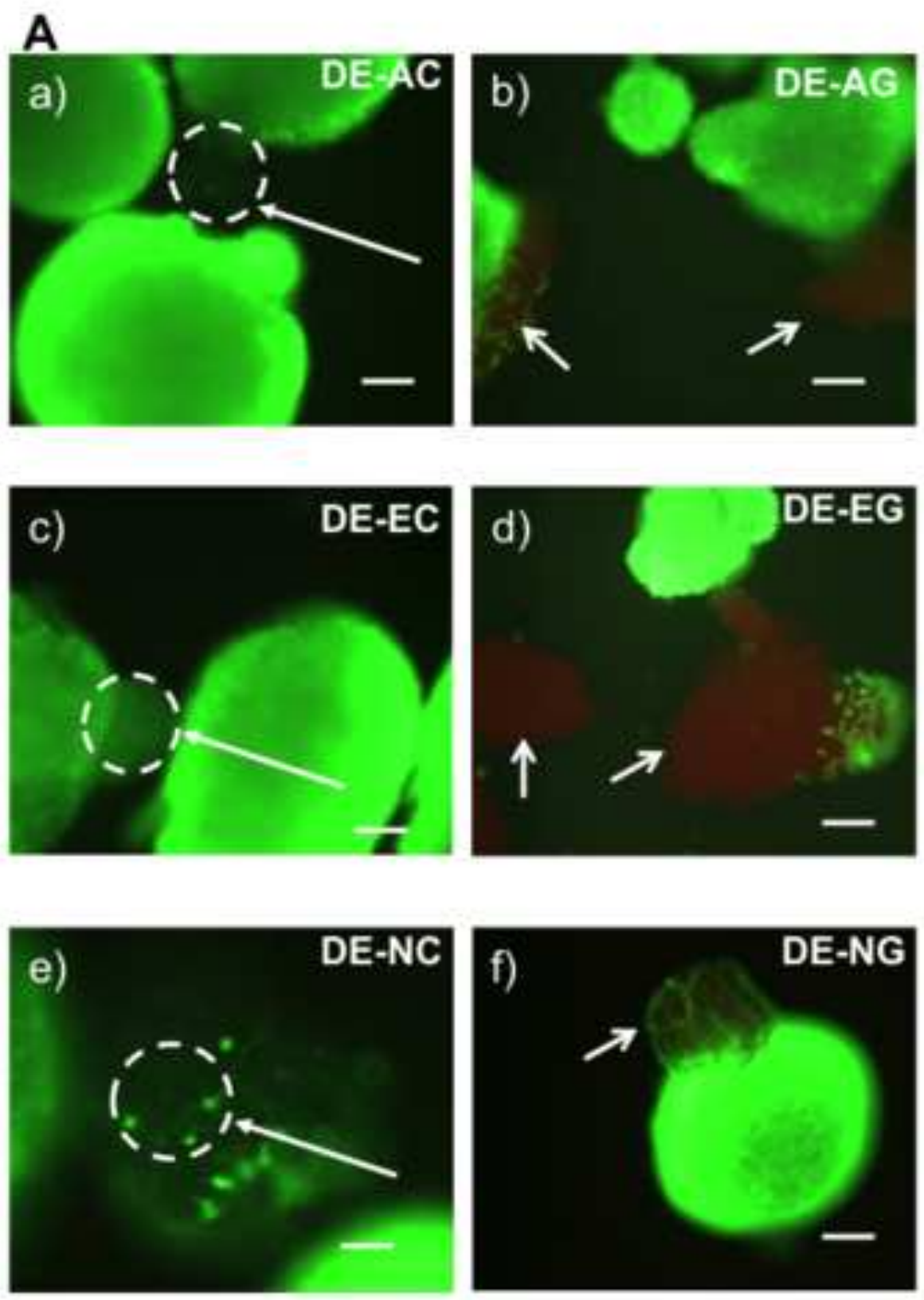
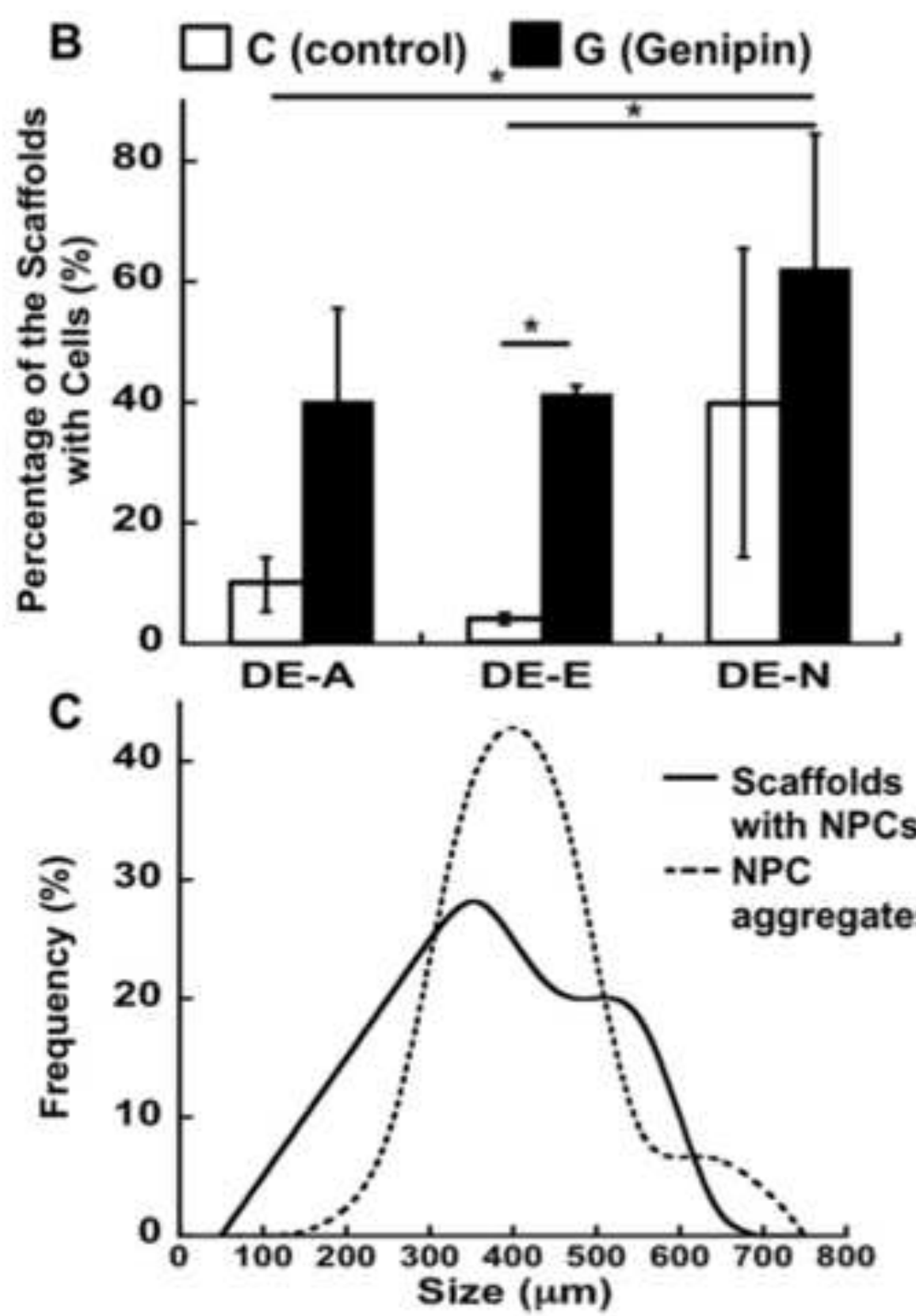
A

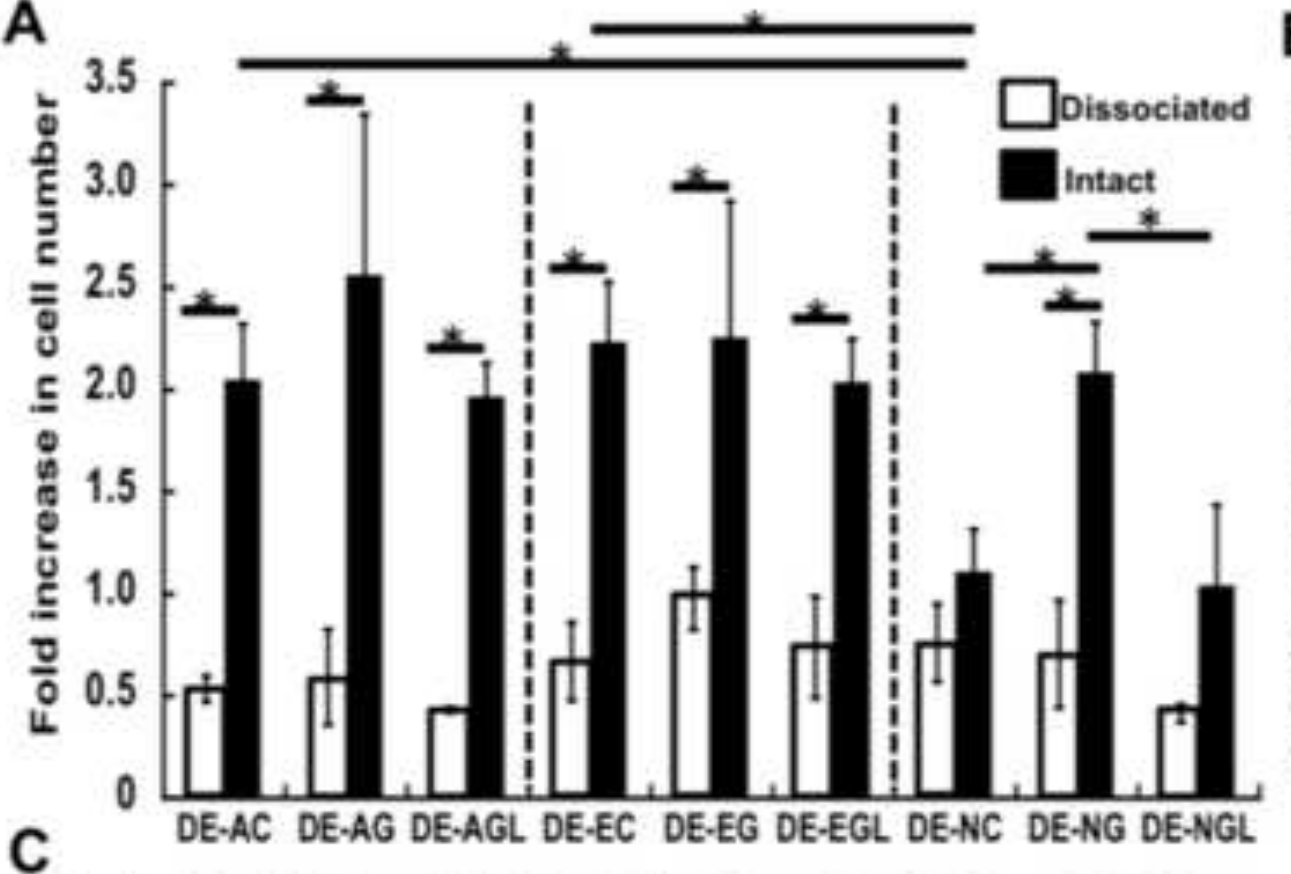

C
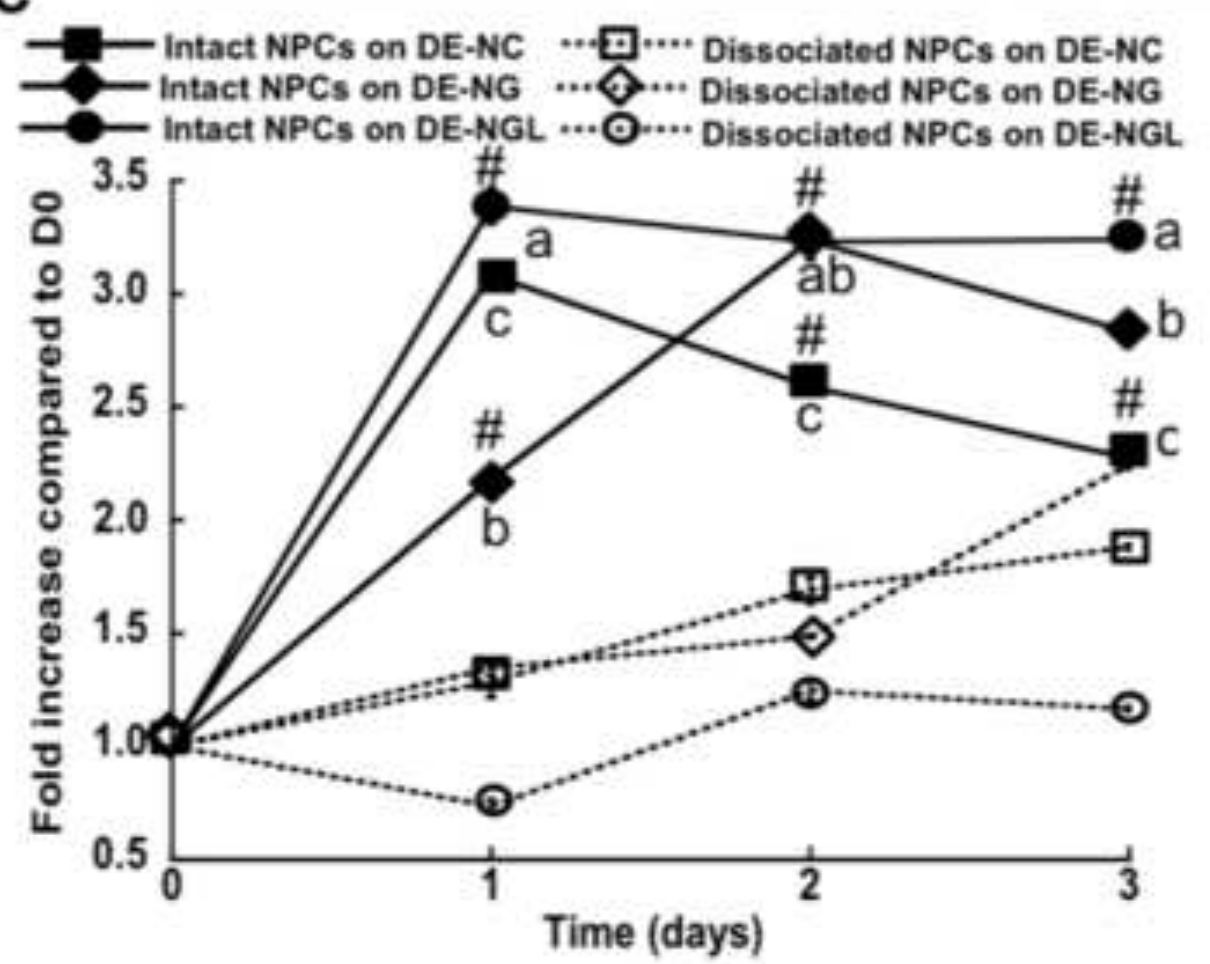

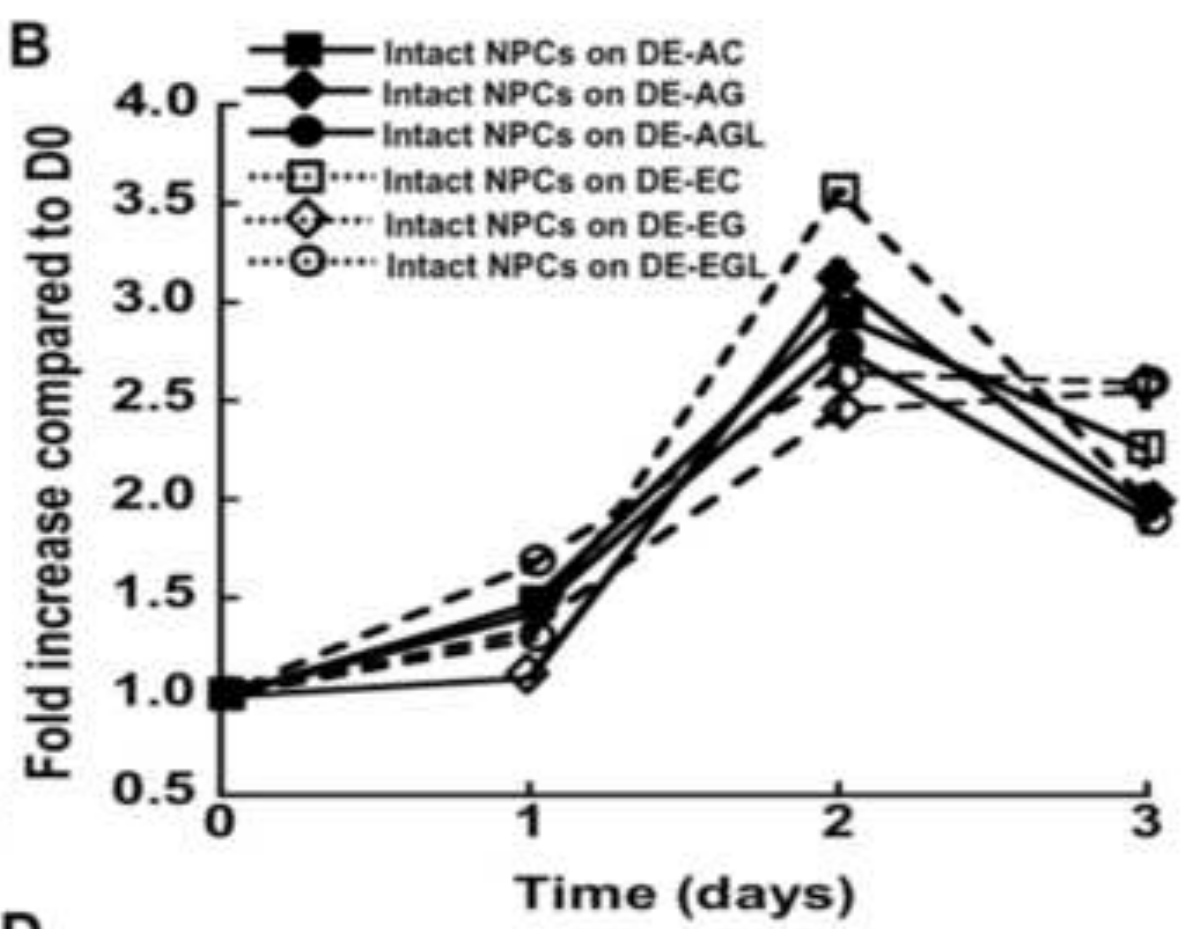

D

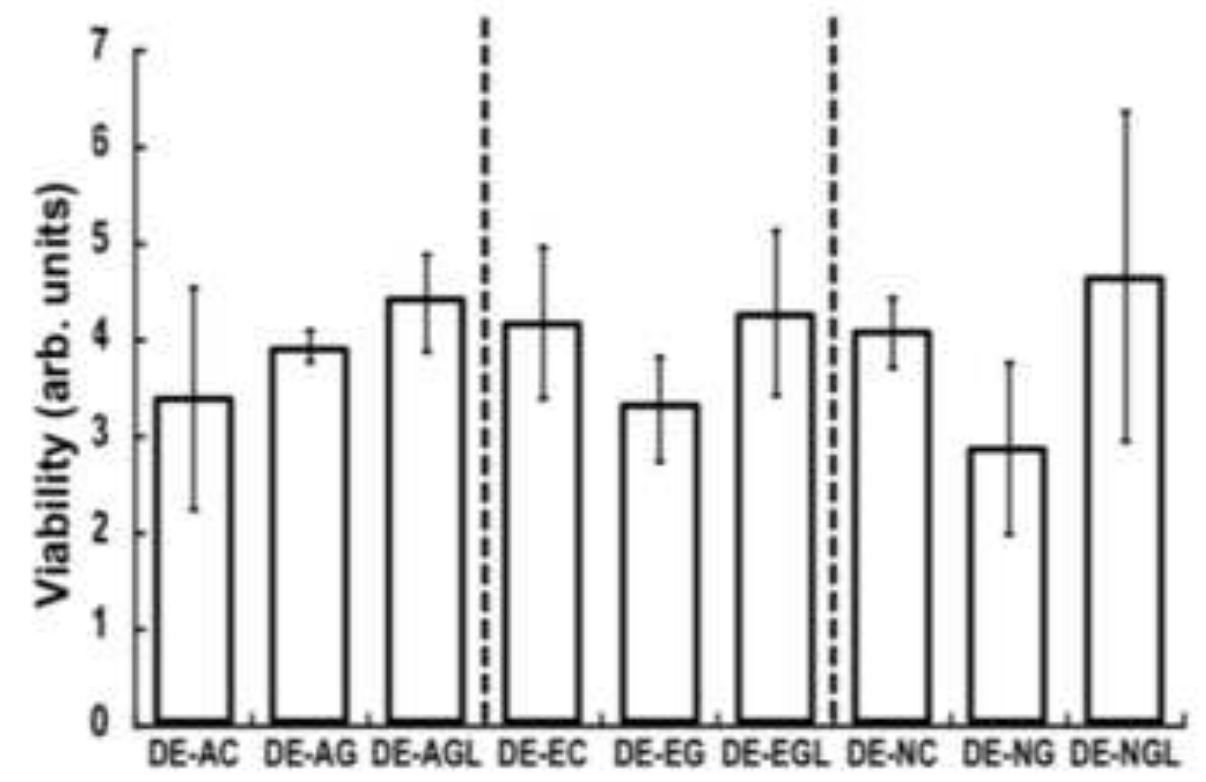




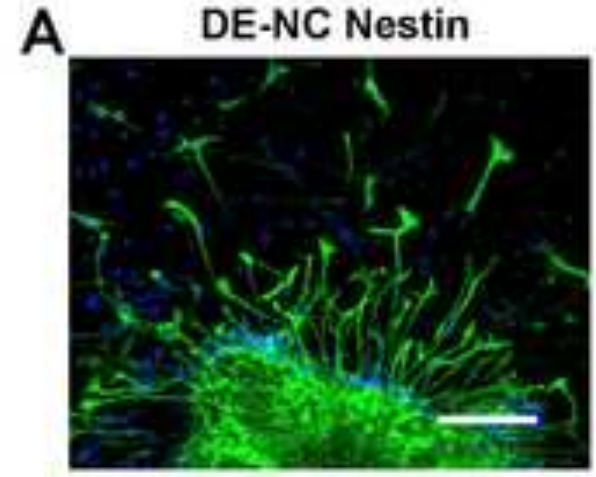

DE-NC Musashi-1
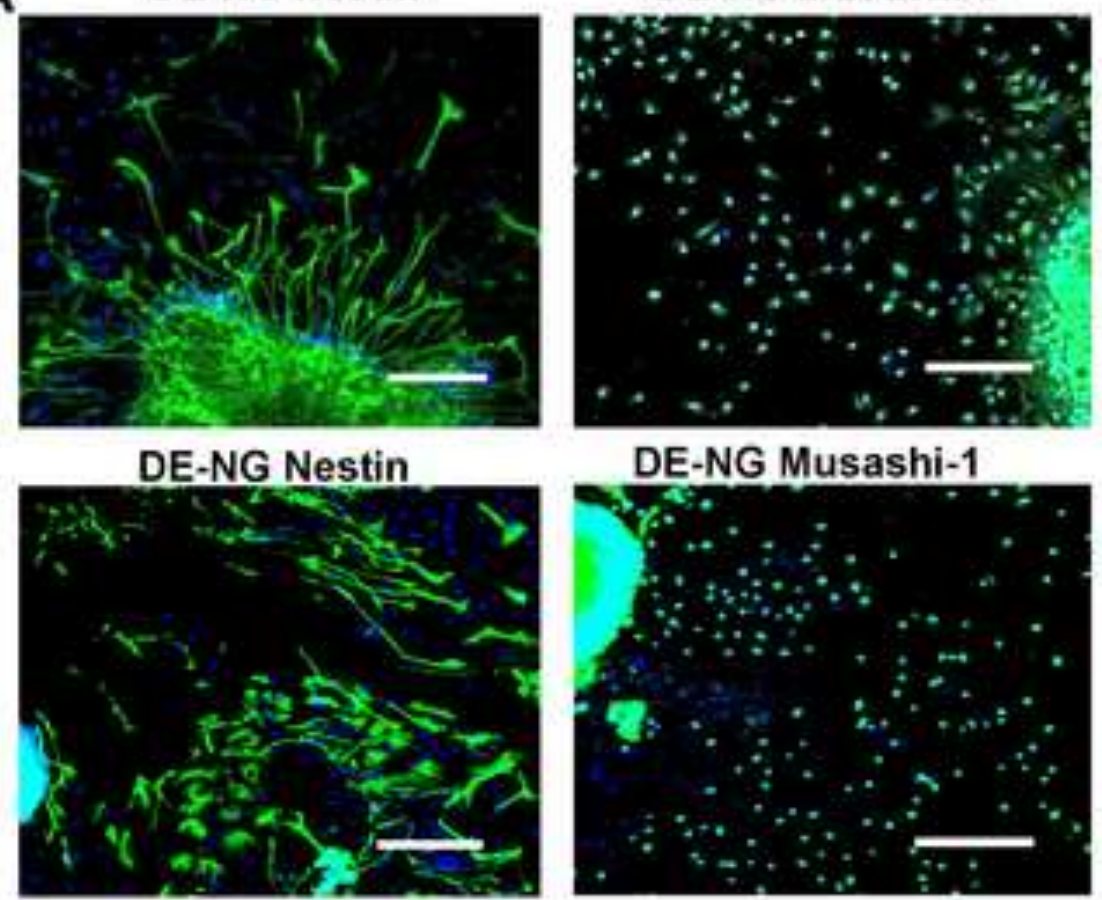

DE-NG Musashi-1

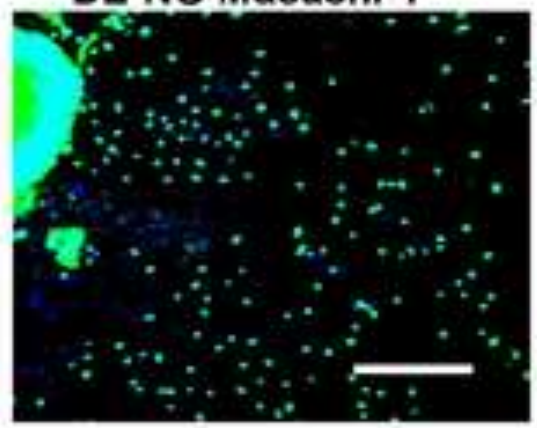

C

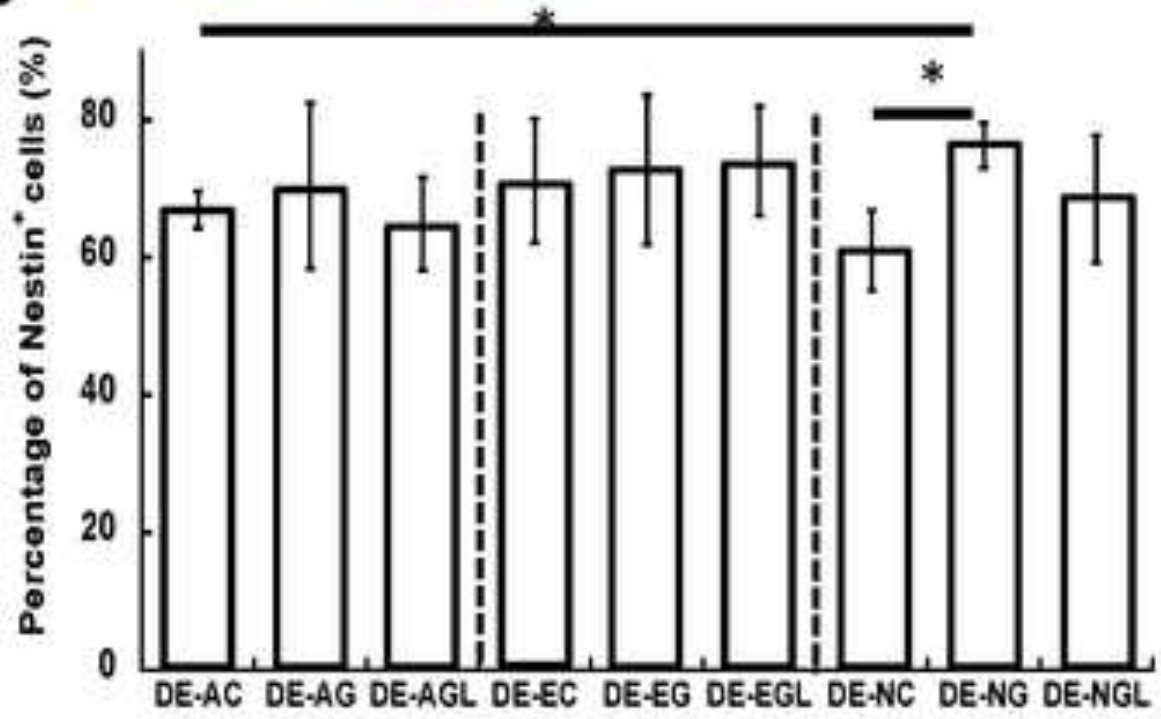

B
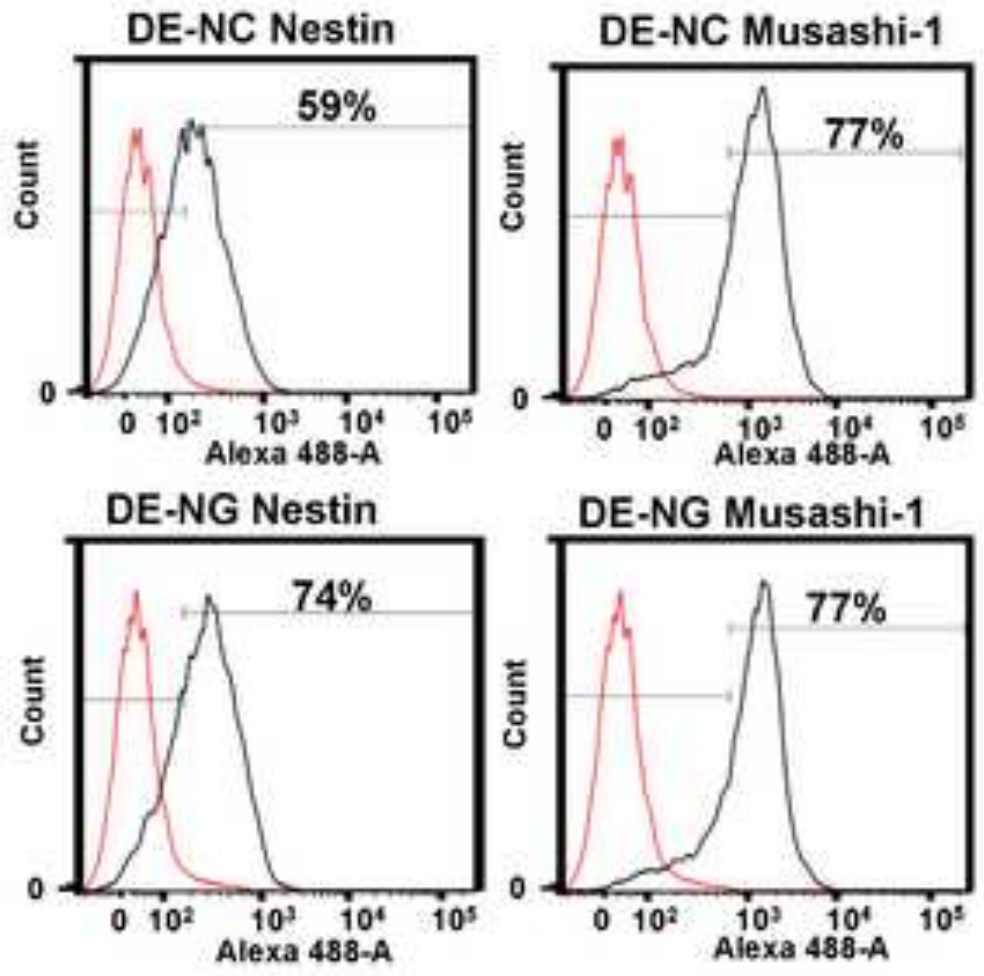

D

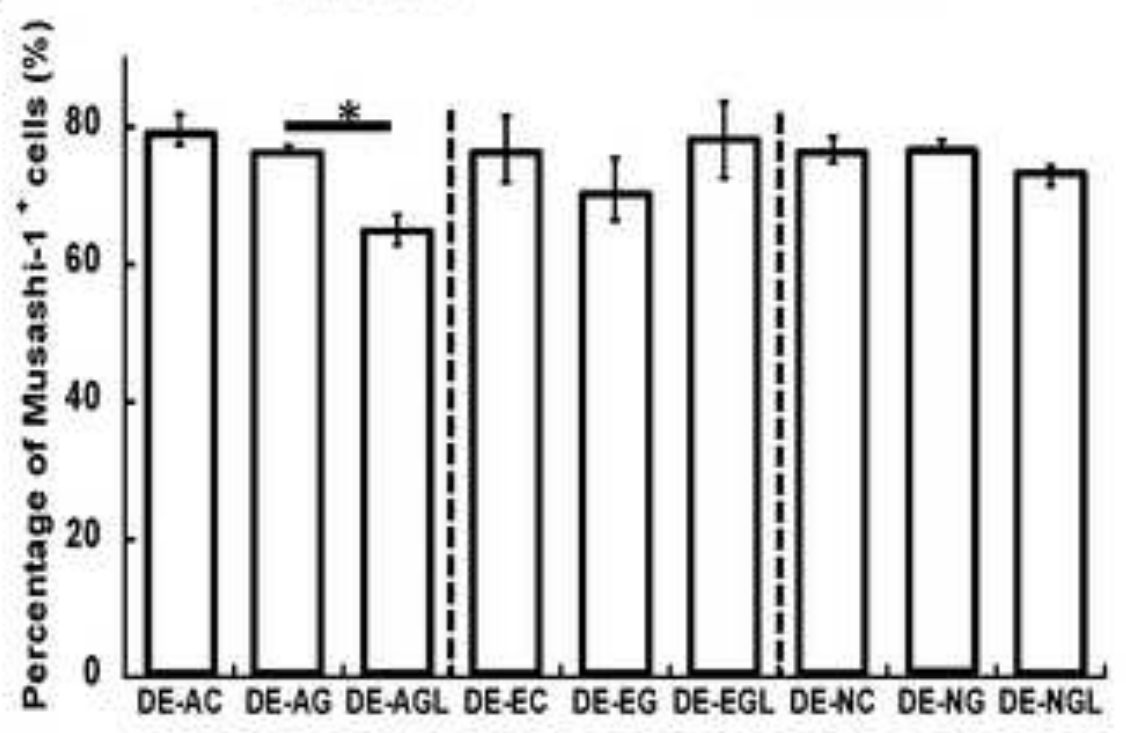


A

DE-NC $\beta$-Tubulin III

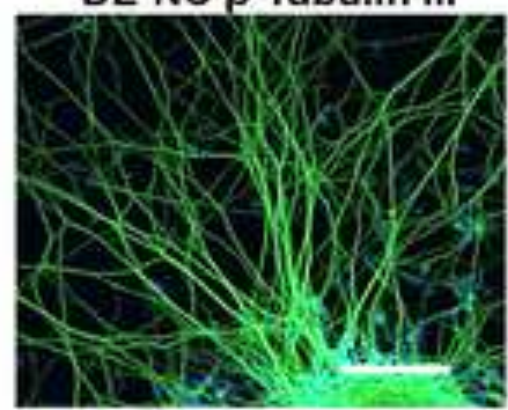

DE-NG $\beta$-Tubulin III

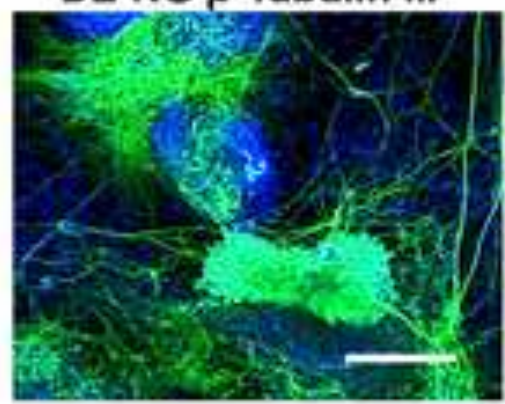

C
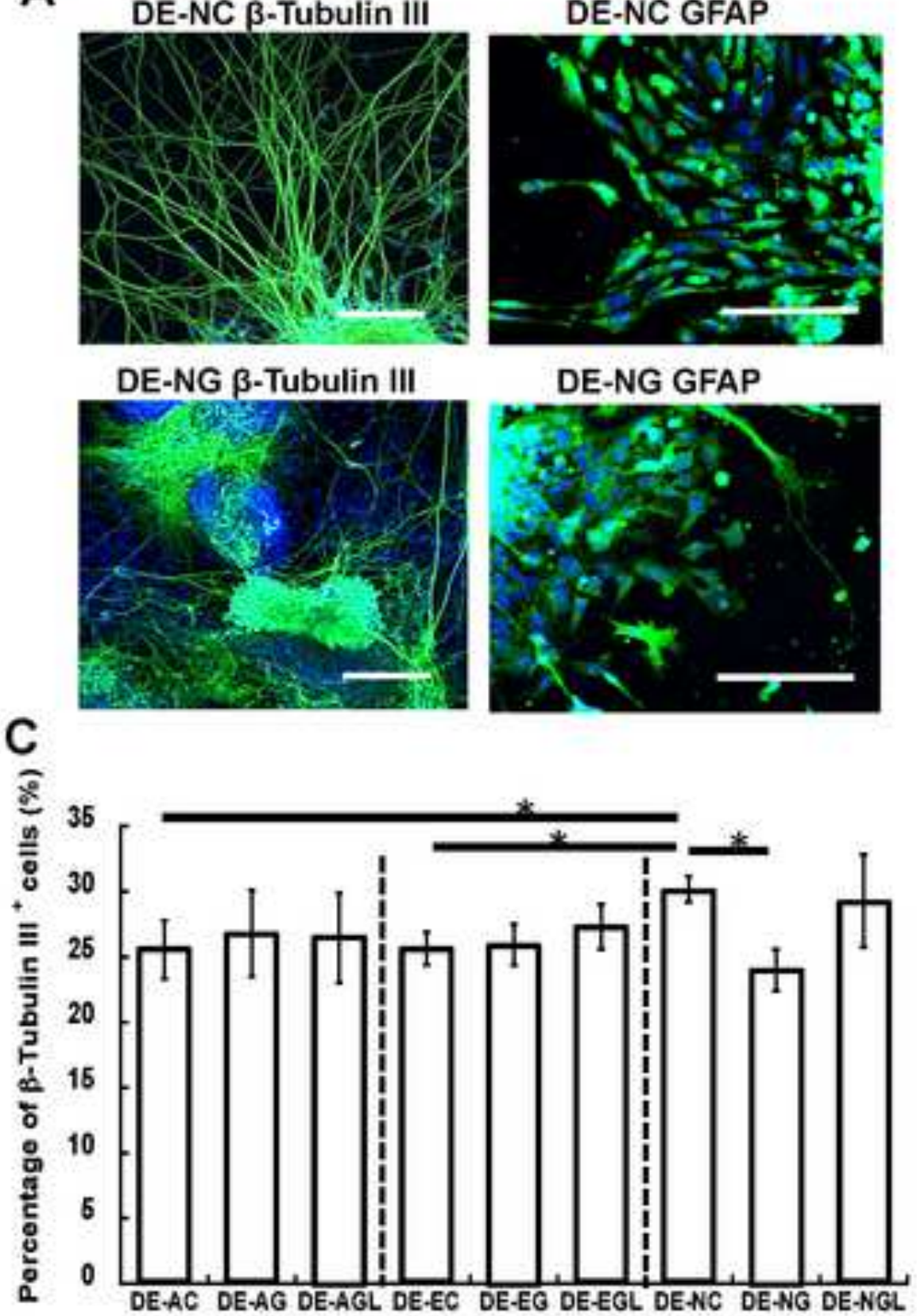

DE-NG GFAP

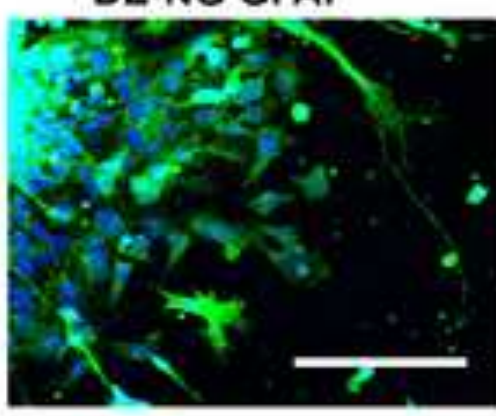

B
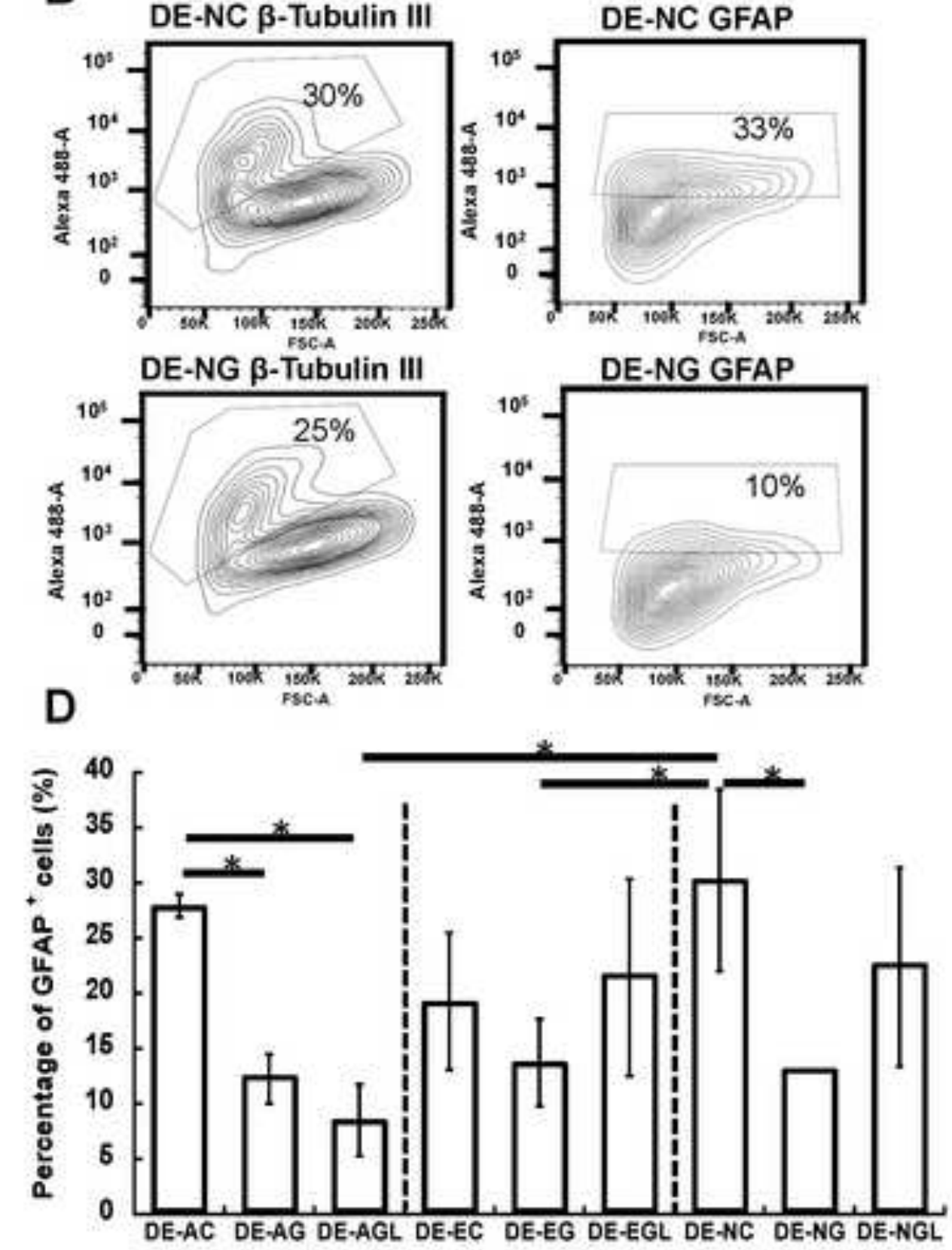

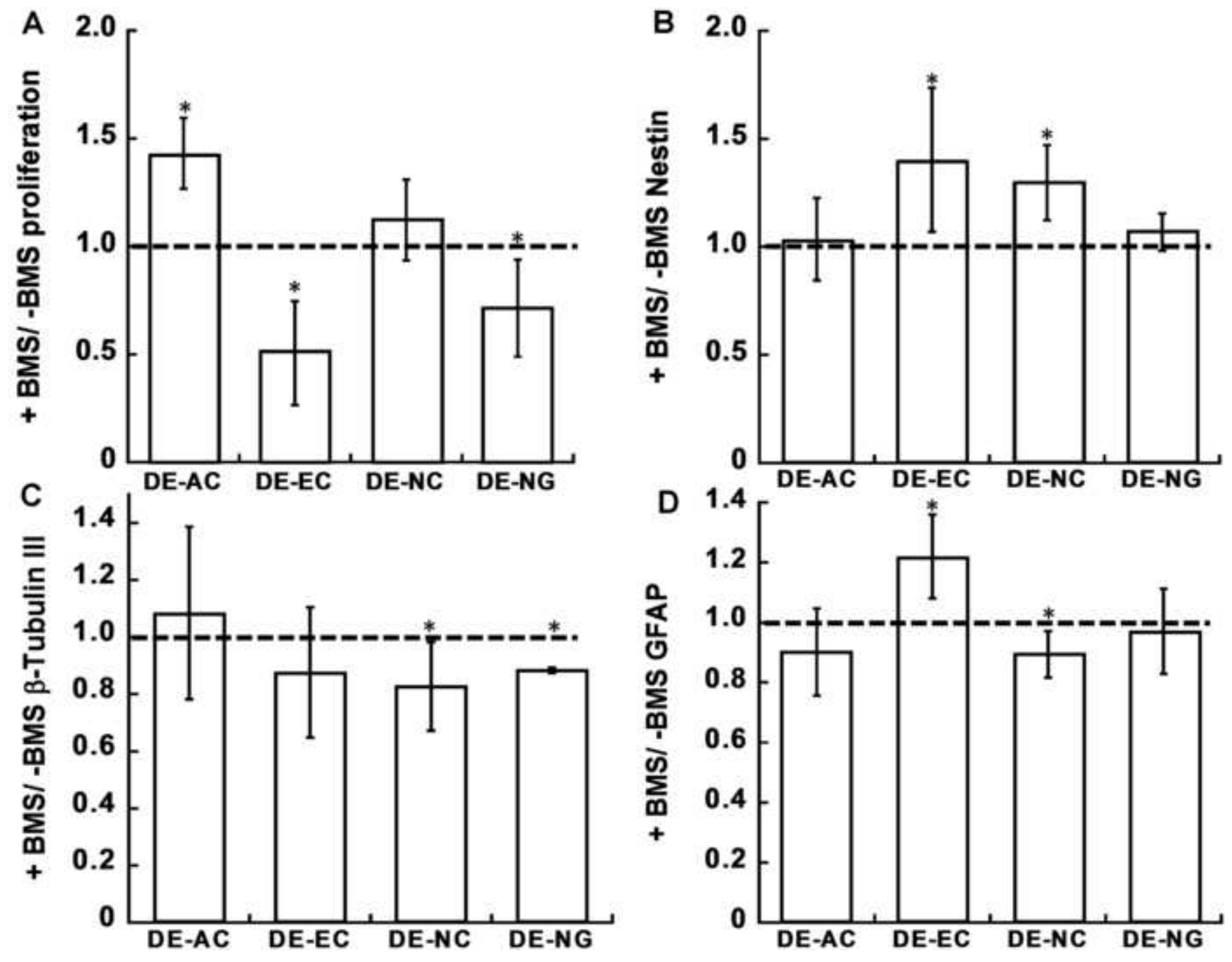